\title{
THE ATMOSPHERE OF SATURN
}

\author{
V. G. TEIFEL \\ Astrophysical Institute, Academy of Sciences Kaz. SSR, Alma-Ata, U.S.S.R.
}

\begin{abstract}
The results of recent studies of the optical properties, temperature, chemical composition and a probable structure of Saturn's atmosphere are reviewed.
\end{abstract}

\section{Introduction}

During recent years great attention has been directed to the study of Jupiter, since this planet is the first object of far space which may be studied in the near future by space methods. It seems a quite understandable tendency to obtain more and more observational data about this planet now, since this information is indispensable for the treatment of the aims and methods of the space researches on Jupiter. Nevertheless much new data were obtained about another major planet - Saturn. These data have shown some sufficient discrepancies between these two greatest bodies of our planetary system.

We shall give here a short review of recent studies of Saturn and of the data on optical properties, chemical composition and probable structure of Saturn's atmosphere.

\section{Atmospheric Composition}

In the atmosphere of Saturn, only methane and molecular hydrogen were discovered by spectral methods. Some indications of the presence of ammonia were derived from spectroscopy but these data are very uncertain and may be rather erroneous. More reliable results were obtained by radioastronomical methods that permit the study of atmospheric layers under the clouds and which have shown the presence of noticeable amounts of ammonia in the lower atmosphere of Saturn. It is necessary to note immediately that the quantitative estimates of the gas abundances, obtained by the spectral measurements of molecular lines and bands, can not be entirely certain because the visible intensity of the absorption features is affected by the multiple scattering in the aerosol medium. Now we shall consider the data on the abundance of some gases derived from the spectral and microwave observations.

\subsection{HYDROGEN}

According to the measurements of Tanaka (1967), the equivalent width of $S(1)$ line in the (3-0) quadrupole band of $\mathbf{H}_{2}$ is $0.078-0.089 \AA$ for the center of Saturn's disk. The equivalent path of $\mathrm{H}_{2}\left(L_{\mathrm{H}_{2}}\right)$ may be obtained as about $70 \mathrm{~km}$-atm (neglecting the effect of the line saturation) or about $330 \mathrm{~km}$-atm (if the line is saturated). For this line Owen (1969) has obtained $W=0.065 \pm 0.010 \AA$ and $L_{\mathrm{H}_{2}} \approx 190 \pm 40 \mathrm{~km}$-atm if the line is saturated. There are no other quantitative data on the $\mathrm{H}_{2}$-abundance derived 
from line intensity measurements. Using the theory of collision narrowing of hydrogen lines developed by Fink and Belton (1969), we can obtain approximately the limits of $L_{\mathrm{H}_{2}}$ from the measurements noted above. Assume for Saturn's atmosphere $T \approx 100 \mathrm{~K}$ and $P_{\text {eff }} \approx 1$ atm. Then we obtain for the S(1) line of $\mathbf{H}_{2}(3-0)$ band the Doppler width $\alpha_{D}=0.0373 \mathrm{~cm}^{-1}$ and the parameter

$$
y=\frac{\alpha_{\mathrm{L}}}{\alpha_{\mathrm{D}}}=1.9 \cdot 10^{-2} \lambda P\left(\frac{T}{T_{0}}\right)^{-1.25}=0.0543,
$$

where $\alpha_{L}$ - the Lorentz width of the absorption line. For the line $S(1)$ in (4-0) band of $\mathrm{H}_{2} \alpha_{\mathrm{D}}=0.0477 \mathrm{~cm}^{-1}$ and $y=0.0425$. Using the values of $y$ and $W / \alpha_{D} \sqrt{\pi}$ we have derived from the curve of growth calculated by Fink and Belton that the value

$$
u=\frac{N s_{0}}{\alpha_{D} \sqrt{\pi}}
$$

(where $N$ - a quantity of absorbing gas, $s_{0}$ - the absorption coefficient for line) is about $8-11$ and $\sim 0.4$ for the $S(1)$ lines of each band consequently. If the effective atmospheric mass in a center of Saturn's disk is assumed $\eta=2$ we have $L_{\mathrm{H}_{2}} \approx 193-$ $165 \mathrm{~km}$-atm from Tanaka's measurements and $L_{\mathrm{H}_{2}} \approx 97 \mathrm{~km}$-atm from the Giver and Spinrad (1966) data for the (4-0) $\mathrm{H}_{2} \mathrm{~S}$ (1) line in Saturn's spectrum. Thus we do not have a very certain result even if we take into account the collision-narrowing effect: the equivalent path of $\mathrm{H}_{2}$ varies from $\sim 100 \mathrm{~km}$-atm to $\sim 260 \mathrm{~km}$-atm for a simple reflecting model of the line formation. The partial pressure of $\mathrm{H}_{2}$ at the base level of a homogeneous layer with that thickness must be $0.8<P<2.2 \mathrm{~atm}$, and it does not contradict the value of $P_{\text {eff }}$ assumed above for our calculations. The values of $L_{\mathrm{H}_{2}}$ noted above were obtained without taking into account the effect of the cloud layer on the formation of absorption lines; these $L$-values can not be accounted as the true $\mathrm{H}_{2}$ abundance in the effective zone of atmosphere where the $\mathrm{H}_{2}$ lines are formed.

There is another way to estimate the $\mathrm{H}_{2}$ abundance in the atmosphere above the clouds. It uses the Rayleigh scattering effects in the ultraviolet spectrum of Saturn. We shall consider the ultraviolet measurements later, in connection with the question of the aerosol layer structure. From the UV measurements of Saturn's spectral reflectivity at $\lambda \lambda 0.33-0.45 \mu$ we have obtained the equivalent thickness of $\mathrm{H}_{2}$, over the aerosol layer, of about $13.5 \pm 1.0 \mathrm{~km}$-atm in the equatorial belt and about $19 \pm 2 \mathrm{~km}$ atm in the temperate latitudes. It is about one order of magnitude less than that derived from the measurements of quadrupole lines.

\subsection{Methane}

From Kuiper's measurements (1947), the equivalent path of $\mathrm{CH}_{4}$ is about $350 \mathrm{~m}$-atm for Saturn. This is probably an overestimate. As we have found from photographic and photoelectric spectrophotometry (Teifel et al., 1971, 1973) the equivalent width of the $0.62 \mu$ absorption band of $\mathrm{CH}_{4}$ in the Saturn spectrum is only about 1.3-1.5 times more than in Jovian spectra. This band may be considered as non- 
saturated. Then if for Jupiter $L_{\mathrm{CH}_{4}} \approx 130 \mathrm{~m}$-atm according to Owen and Mason (1969) we have for Saturn's equatorial belt $L_{\mathrm{CH}_{4}} \approx 170 \mathrm{~m}$-atm and for the south temperate belt $L_{\mathrm{CH}_{4}} \approx 200 \mathrm{~m}$-atm. The same results were obtained from comparison of the intensity of the $0.543 \mu \mathrm{CH}_{4}$ band in the spectra of Saturn and Jupiter scanned by a photoelectric spectrometer (Bugaenko et al., 1971). These values of $L_{\mathbf{C H}_{4}}$ are the upper limits because multiple scattering must increase the intensity of the weak absorption bands. As will be shown later, the most probable upper limit of the $\mathrm{CH}_{4}$ relative abundance on Saturn is about $3.8 \times 10^{-3}$, if the scattering effects are taken into account.

\subsection{Ammonia}

The question of the presence of ammonia in Saturn's atmosphere is very important in connection with the problem of the cloud composition. The physical conditions in the atmosphere of this planet are favourable for ammonia sublimation and the formation of ammonia clouds.

The spectroscopic observations have not detected a more intensive (than in visible)

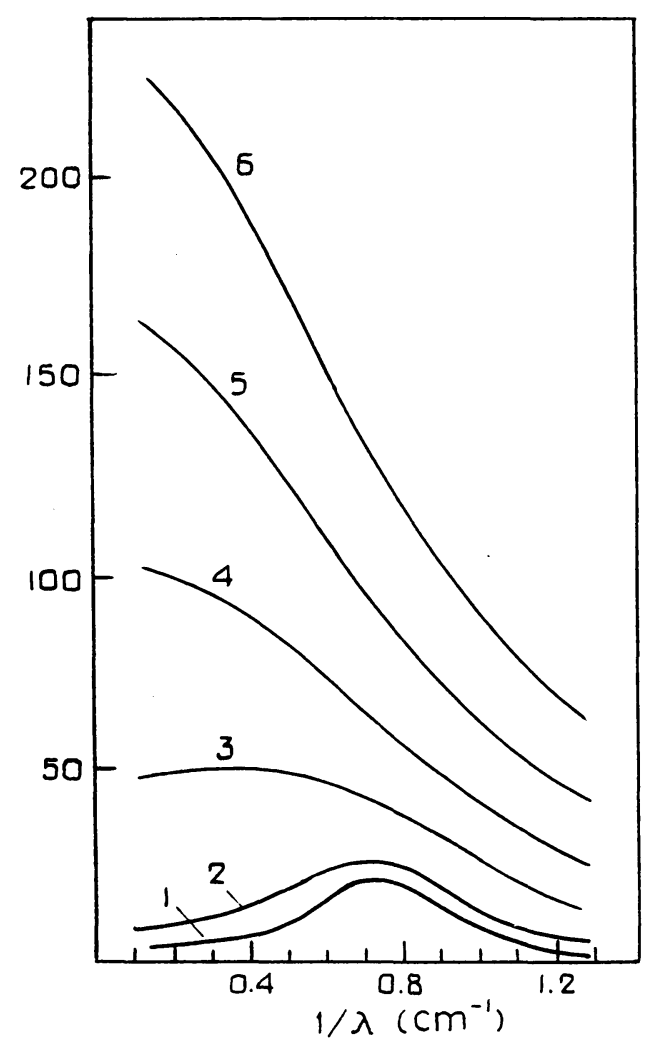

Fig. 1. The absorption coefficients of $\mathrm{NH}_{3}$, in a hydrogen atmosphere at $T=293 \mathrm{~K}$, at the pressures of $7 \mathrm{~atm}$ (1), $10 \mathrm{~atm}$ (2), $20 \mathrm{~atm}$ (3), $30 \mathrm{~atm}$ (4), $40 \mathrm{~atm}$ (5) and $50 \mathrm{~atm}$ (6), after Kuzmin et al. (1972). 


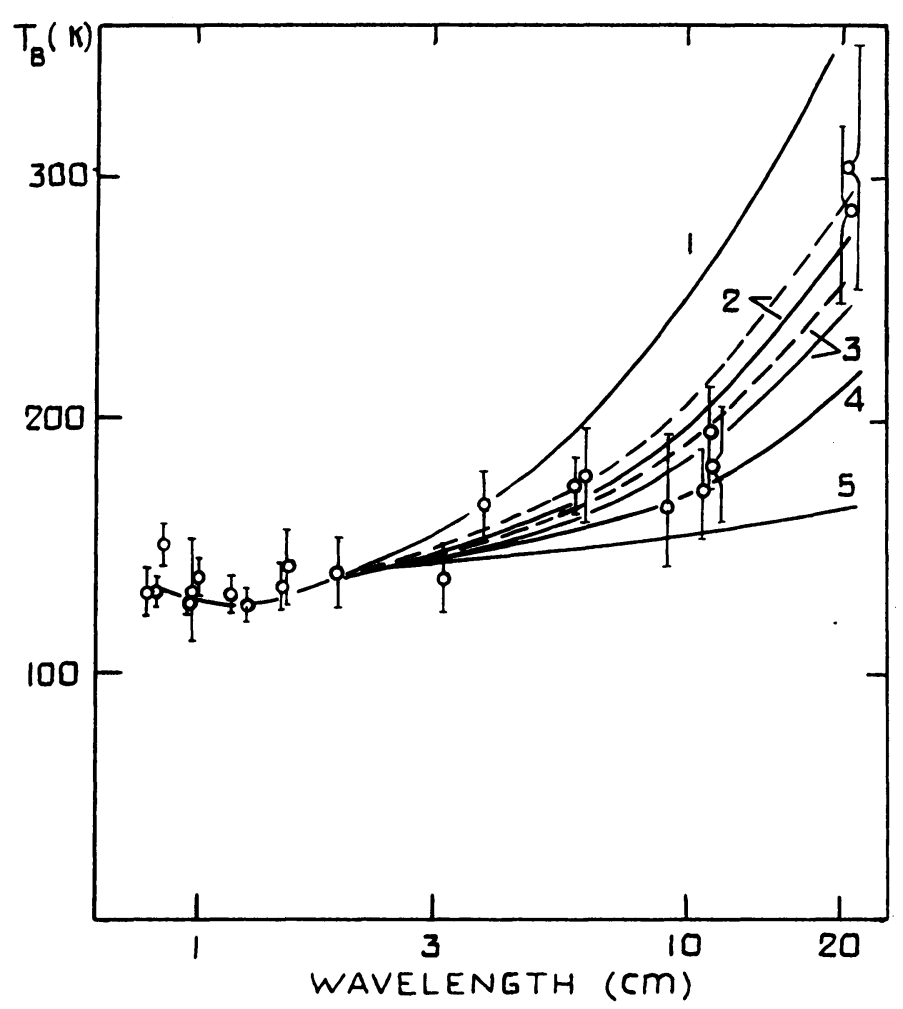

Fig. 2. A comparison of the calculated and observed spectral variations of the brightness temperature of Saturn. Solid lines - a pure hydrogen atmosphere, dotted lines - a hydrogen-helium mixture. The relative abundances of $\mathrm{NH}_{3}$ in the undercloud atmosphere are $10^{-5}(1), 3 \times 10^{-5}$ (2), $5 \times 10^{-5}(3), 10^{-4}(4), 10^{-3}(5)$. After Kuzmin et al. (1972).

infrared ammonia band at $\lambda 1.51 \mu$. Moroz (1966) has estimated an upper limit to the ammonia abundance of about $50 \mathrm{~cm}$-atm, from the absence of this band in his scans of Saturn's spectrum. Wrikson and Welch (1970) have concluded from the study of millimeter radio emission from Saturn that the quantity of $\mathrm{NH}_{3}$ above the level with $T 125-130 \mathrm{~K}$ can not be more than about $8 \mathrm{~cm}$-atm; hence it is natural that the spectral observations have not detected ammonia absorption in the optical spectrum.

Kuzmin et al. (1972) have calculated theoretically the spectral distribution of the brightness temperature in the microwave spectrum of Saturn for different relative abundances of $\mathrm{NH}_{3}$, taking into consideration the influence of low and high pressures on the absorption coefficients of ammonia. These absorption coefficients were derived for the levels with low pressures $(P<6.5 \mathrm{~atm})$ anf for the deep layers of atmosphere $(P>6.5 \mathrm{~atm})$ separately. Values of the $\mathrm{NH}_{3}$ absorption coefficients vs wavelength are shown for some pressures in Figure 1. The best agreement between the calculated dependence of $T$ and $\lambda$ with the radio observations may be noted at a relative ammonia abundance of about $(3-5) \times 10^{-5}$ in the undercloud atmosphere of Saturn (Figure 2). It is interesting that the radio emission at $\lambda>30 \mathrm{~cm}$ in this case must be 
formed in very deep atmospheric layers where the pressure may be more than $1000 \mathrm{~atm}$. In this region multiple molecular collisions must take place and therefore the study of the molecular absorption at very high pressures is necessary.

\subsection{Helium}

The relative abundances of hydrogen and helium are the fundamental characteristics of the atmospheres of major planets, since these gases determine the mean molecular weight and thermodynamic properties of atmospheres. Unfortunately, even for Jupiter the ratio $\mathrm{H}_{2}$ : He is not known with a sufficient accuracy. According to estimates of Owen and Mason (1969), the most probable value of $\mathrm{H}_{2}$ : He may be about 5:1 for the number of molecules. This ratio was derived from measurements of the methane lines halfwidths, which are chiefly determined by collisions with $\mathrm{H}_{2}$ and $\mathrm{He}$ molecules. It is not improbable that the relative abundance of helium may be somewhat less than 0.2 if some part of the line broadening is caused by multiple scattering effect in the aerosol medium where the weak methane lines are formed. There are no halfwidth measurements for the methane lines in Saturn's spectrum and we only speak about the relative helium abundance in Saturn's atmosphere by the analogy with Jupiter.

\section{Temperature}

After the first radiometric measurements published by Menzel et al. (1926), no further investigations of the thermal emission of Saturn were performed for a very long time. The most important results from recent experiments have been obtained by Aumann et al. (1969). Their measurements, at a wide range of wavelengths (from 1.5 to $350 \mu$ ), have made it possible to estimate the entire flux of the thermal emission of Saturn (about $8.7 \times 10^{-13} \mathrm{~W} \mathrm{~cm}^{-2}$ ) and the effective temperature of planet $T_{e}=97 \pm 4 \mathrm{~K}$. This temperature is significantly more than the equilibrium temperature, which is about $77 \mathrm{~K}$ if planetary albedo $A_{v} \approx 0.45$. Thus the heat flux of Saturn is about 2.5 times more than may be due to heating by the solar radiation only. It means that Saturn, like Jupiter, has it's own internal heat sources. The most probable source may be a gravitational contraction of the planet. The heat flux may be carried by convection in the planet interiors.

The temperatures derived from measurements in different parts of the infrared spectrum are not different in principle from $T_{e}$ but they are somewhat lower than the temperatures obtained by the earliest observations. Now there are many estimates of the brightness temperature of Saturn in the microwave range - from 12 to $50 \mathrm{~cm}$. These estimates give higher means of $T_{B}$ (Figure 3 ) than those derived from optical observations. The temperature increases with the wavelength, but not as sharply as is observed for Jupiter, where at $\lambda \geqslant 3 \mathrm{~cm}$ a significant role is played by nonthermal emission. The microwave spectrum of Saturn may be interpreted solely as the thermal radiation output from depths which increase with wavelength: the brightness temperature of Saturn at $\lambda 49.5 \mathrm{~cm}$ is about $390 \pm 65 \mathrm{~K}$ (Yerbury et al., 1971) and it is about $540 \pm 110 \mathrm{~K}$ at $\lambda 94.3 \mathrm{~cm}$ (Yerbury et al., 1973). There are no certain data on 


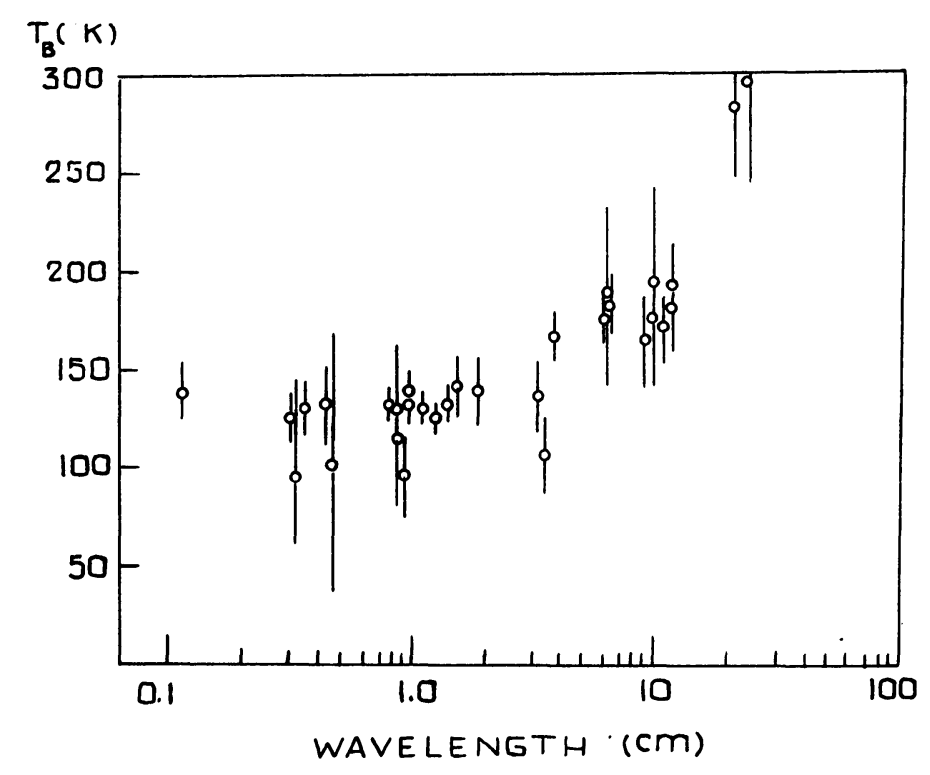

Fig. 3. The spectrum of the microwave emission of Saturn.

the presence of linear polarization of Saturn's radio emission, which might have been considered as evidence of a nonthermal component in the microwave radiation. The interferometric measurements at $\lambda 11 \mathrm{~cm}$ (Kellermann, 1966; Berge and Read, 1968) have shown that practically the entire radio emission is formed in a region whose angular dimensions are not more than the visible disk of planet. This means that there are no radiating belts around Saturn. The presence of significant magnetic field near Saturn is also scarcely probable.

\section{TABLE I}

Temperature of Saturn from the optical measurements

\begin{tabular}{cllc}
$\begin{array}{l}\text { Wavelengths, } \\
\text { microns }\end{array}$ & $T(\mathrm{~K})$ & Author & Notes \\
\cline { 1 - 3 } $8-14$ & 125 & Menzel et al. (1926) & $T_{B}$ \\
$8-14$ & $85 \pm 2$ & Low (1964) & $T_{B}$ \\
$8-14$ & $93 \pm 3$ & Low (1964) & $T_{B}$ \\
$8-14$ & 100 & Murray and Wildey (1963) & $T_{B}$ \\
$8-14$ & $107(+5,-10)$ & Moroz et al. (1968) & $T_{B}$ \\
$10-14$ & $98.3 \pm 3$ & Allen and Murdock (1971) & $T_{B}$ \\
$10-14$ & $99.7 \pm 3$ & Allen and Murdock (1971) & $T_{B}$ \\
5 & 120 & Low and Davidson (1968) & $T_{B}$ \\
20 & $97 \pm 2$ & Murphy (1973) & $T_{B}$ \\
$1.5-350$ & $97 \pm 4$ & Aumann et al. (1969) & $T_{e}$ \\
0.827 & 88 & Spinrad (1964) & $T_{\text {rot }}$ \\
0.643 & $126 \pm 30$ & Giver and Spinrad (1966) & $T_{\text {rot }}$ \\
0.643 & $103 \pm 20$ & &
\end{tabular}


The optical measurements of Saturn's temperature are summarized in Table I along with estimates of the rotational temperatures which were obtained by Giver and Spinrad (1966) from the ratio of the hydrogen quadrupole lines $S(1)$ and $S(0)$ intensities in the (4-0) band and by Spinrad (1964) from measurements of the same lines in the (3-0) band.

It is very interesting that there has not been observed on Saturn the anomalously high brightness temperature near $\lambda 5 \mu$ which was discovered on Jupiter. In some regions of the Jovian disk, especially in the North Equatorial Belt, the brightness temperature at $\lambda 5 \mu$ was about $230 \mathrm{~K}$ or more, according to the observations of Westphal (1969), and Keay et al. (1972). This high temperature may be caused by a decrease in the screening of the thermal radiation by the lower clouds whose density is not homogeneous in the different regions of Jupiter. On Saturn, as can be seen from Table I, the temperature at $5 \mu$ is not much higher than the effective temperature and there are no peculiarities in the temperature distribution on Saturn's disk (Low and Davidson, 1969; Westphal, 1971). It is very hard to suspect the presence of very strong absorption by gases in this spectral range and it is more probable to suggest the presence of a dense lower cloud layer which is opaque for $5-\mu$ emission. Additional researches are very much needed, especially the study of the spectral variations of Saturn's brightness temperature in the infrared.

\section{Optical Properties of the Cloud Layer and Outer Atmosphere}

We now have some definite information about the spectral reflectivity of Saturn from the photoelectric measurements by McCord et al. (1971), Teifel et al. (1971), Irvine and Lane (1971), and Bugaenko (1972). The reflectance of planet in the spectral region outside of the methane absorption bands (at $\lambda<0.7 \mu$ ) is decreased toward the short wavelengths (Figure 4). The most sharp decreasing of reflectivity begins from $\lambda \approx 0.5 \mu$

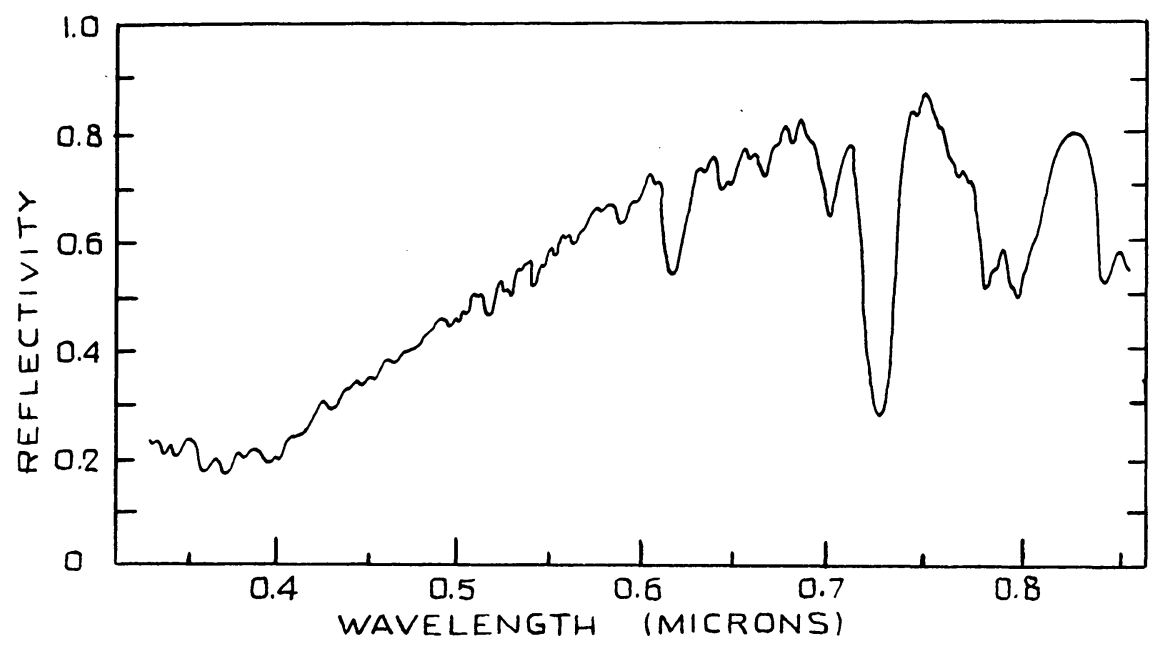

Fig. 4. The absolute spectral reflectivity at the center of Saturn's disk, after Bugaenko (1972). 
to $\lambda \approx 0.38 \mu$, but at $\lambda<0.38 \mu$ the reflectivity is observed to increase and this increase is continued to about $0.25 \mu$, as was determined by ground measurements (Teifel and Kharitonova, 1972a, 1973; McCord et al., 1971; Krugov, 1972) and by OAO observations (Wallace et al., 1972). It might be supposed that the reflectivity depression near $\lambda 0.38 \mu$ is connected with a wide range absorption band of an aerosol. The short wavelength decrease of the reflectivity is caused undoubtedly by the aerosol continuum absorption but the growth of reflectivity at $\lambda<0.38 \mu$ is of other nature. If the aerosol absorption is decreased and the single scattering albedo

$$
\omega_{c}=\frac{\sigma_{a}}{\sigma_{a}+\kappa_{a}}
$$

(where $\sigma_{a}$ is the volume scattering coefficient and $\kappa_{a}$ is the volume coefficient of true absorption) is increased accordingly we must observe at $\lambda<0.38 \mu$ more and more darkening of Saturn's disk toward the limb; this follows from the theory of diffuse reflection of radiation by a scattering medium. However, there is observed a brightening of Saturn's disk near the limb in the ultraviolet (at $\lambda \approx 0.36 \mu$ ) as seen from the photographs of Saturn obtained by Marin (1968), Reese (1971) and others. Also there

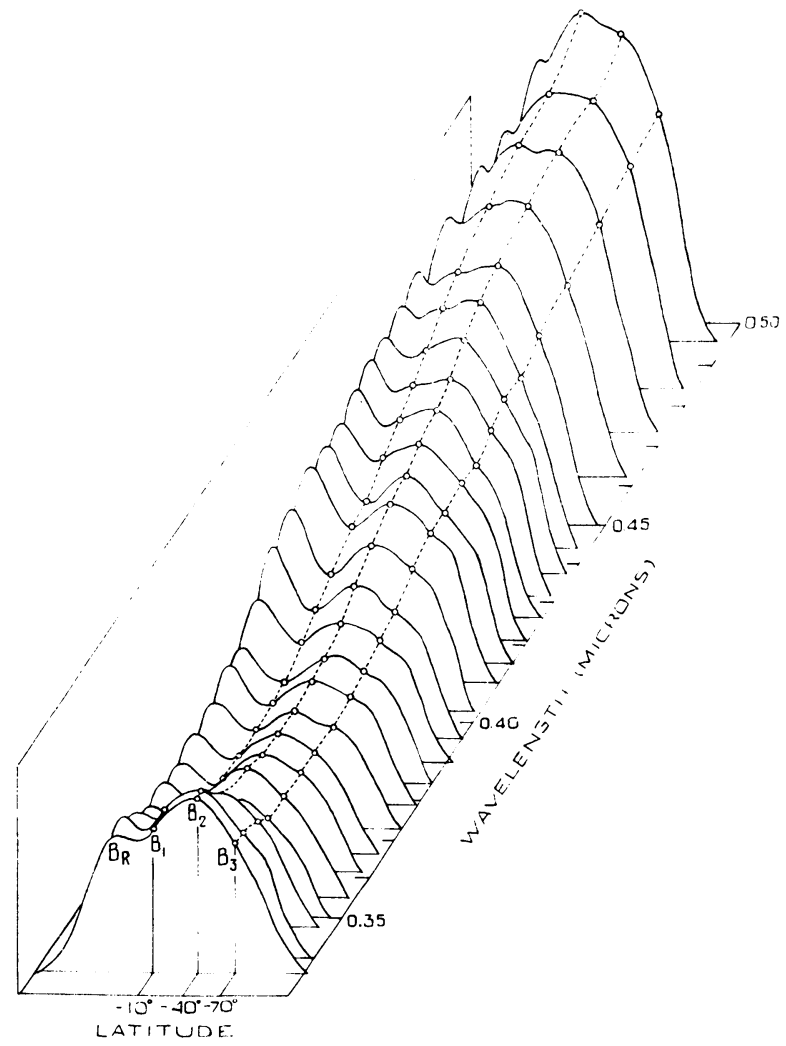

Fig. 5. Spectral variations of photometric monochromatic profiles at the central meridian of Saturn, from photoelectric spectral measurements in 1972 at $\lambda \lambda 0.34-0.50 \mu$. 

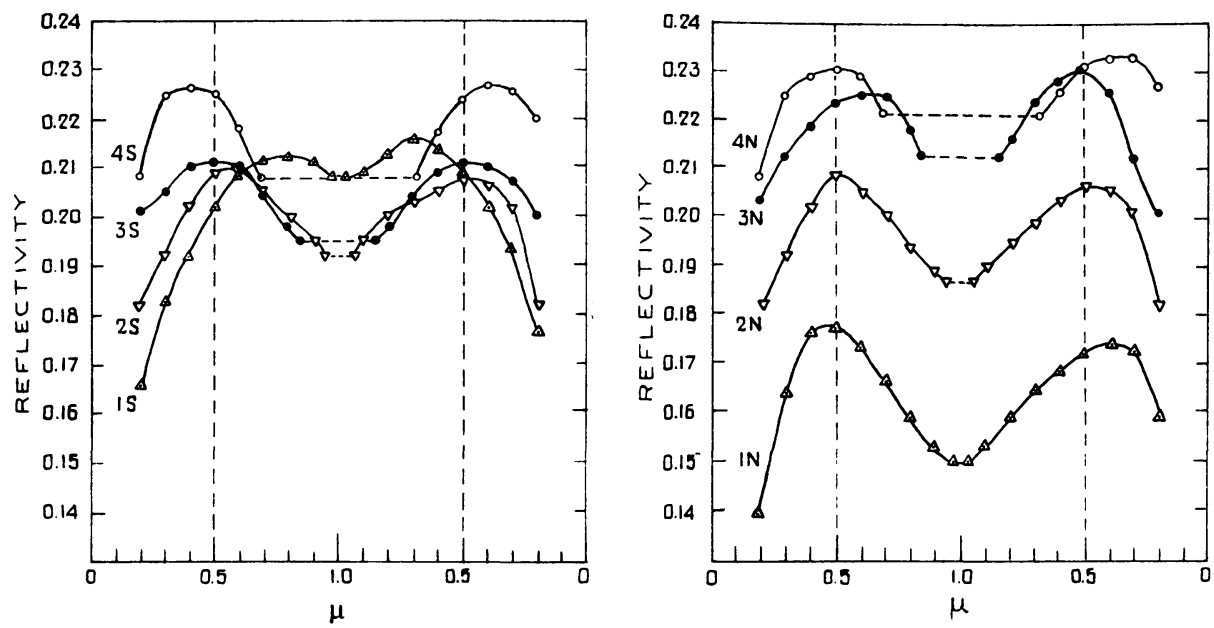

Fig. 6. Variations of reflectivity $r(\mu)$ in some zones of Saturn in 1966, at $\lambda_{\text {eff }}=0.355 \mu$, derived from the observations of Marin (1968).

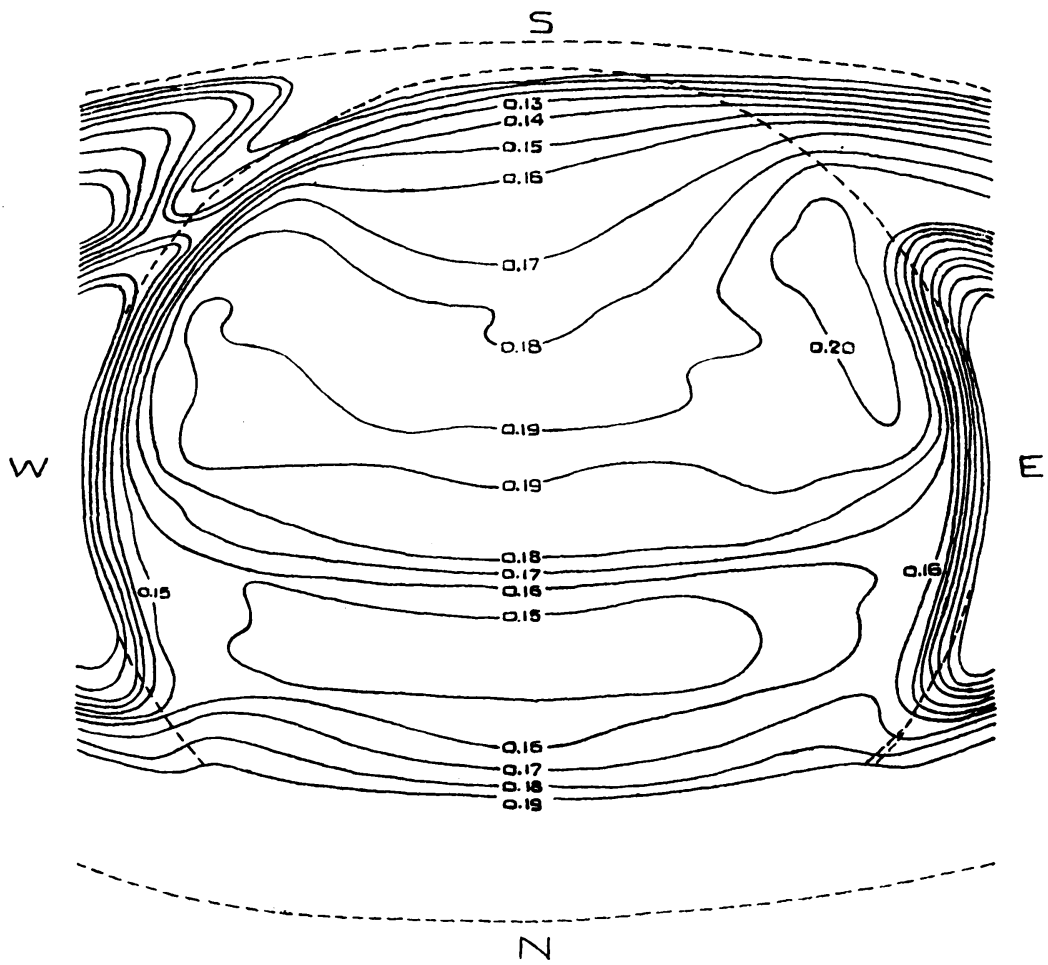

Fig. 7. The isophotes of reflectivity on Saturn's disk in the ultraviolet $\left(\lambda_{\text {eff }}=0.36 \mu\right)$ derived from the measurements of negatives obtained at Mauna Kea Observatory in 1971. 


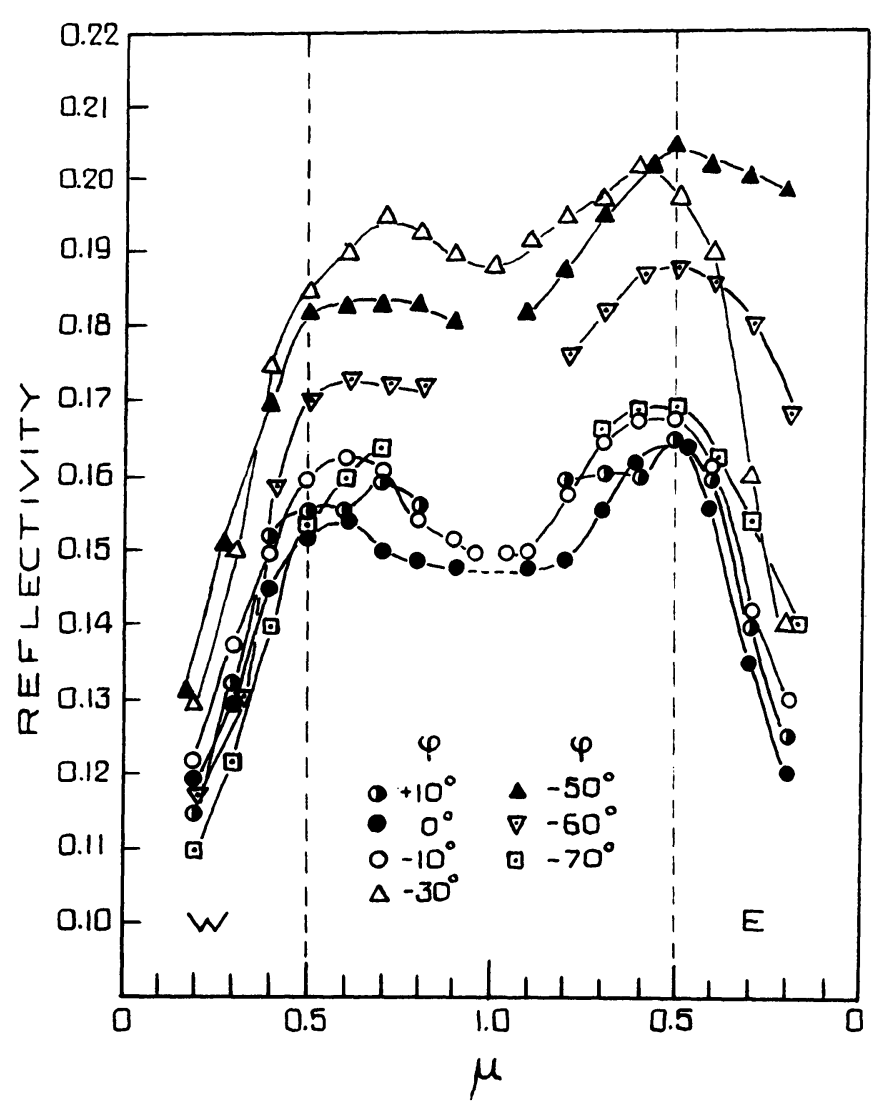

Fig. 8. Variations of reflectivity $r(\mu)$ in the ultraviolet along some saturnographic latitudes $(\varphi)$, derived from the preceding figure.

is other peculiarity: the equatorial belt of Saturn, which was light in the visible, turns into most dark belt on the disk in the ultraviolet light (Figure 5). Since Saturn is observed at different angles of the equatorial plane to the line of sight, the comparison of the reflective properties of any planetary zones will be more reliable if carried out for points falling at the identical conditions of illumination and observation, i.e. at the equal means of $\mu_{0}=\cos i$ and $\mu=\cos \varepsilon$.

We have used the photometric measurements carried out by Marin (1968) from the photographs obtained by him in 1966, when the equator of Saturn was in the Earth-Saturn-Sun plane, and the film photographs of Saturn obtained in 1971 at Mauna Kea Observatory (Hawaii) and kindly offered to us by Dr Cruikshank. For these images we have calculated the values of $\mu$ and planetographic latitudes $\varphi$ using Schoenberg's formulaes (Schoenberg, 1929) and then we have plotted the visible ultraviolet reflectivity $r$ vs $\mu$ for different belts of Saturn. It was assumed from the photoelectric spectrophotometry that $r=0.15$ at $\lambda 0.36 \mu$ in the equatorial belt. The results have shown (Figures 6-8) an increase of reflectivity with latitude, excluding the polar 
regions: the south polar region is more similar to the equatorial belt in ultraviolet. The limb brightening is observed at all latitudes, having maximum of reflectivity near $\mu \approx 0.5-0.6$. The displacement of the maximum from the limb is caused by turbulence of the Earth's atmosphere; however the increasing of brightness toward the limb on Saturn's disk in the ultraviolet may be interpreted as a result of Rayleigh scattering in the overcloud gaseous atmosphere. A theoretical study of Rayleigh scattering in planetary atmospheres (Coulson et al., 1960) has given the brightness of a Rayleigh atmosphere increasing up to limb, but in the presence of atmospheric turbulence the visible intensity distribution is distorted and may be given in the one-dimensional case by the formula

$$
f(x)=\frac{1}{\sigma \sqrt{ } 2 \pi} \int_{-1}^{+1} F\left(x^{\prime}\right) \exp \left[-\frac{\left(x-x^{\prime}\right)^{2}}{2 \sigma^{2}}\right] \mathrm{d} x^{\prime},
$$

where $F\left(x^{\prime}\right)$ is the true distribution of the brightness along the radius (axe $x$ ) and $\sigma$ is a parameter of the Gauss curve which describes the distribution function for the star image oscillation. If $F\left(x^{\prime}\right)$ is the theoretical variation of the Rayleigh atmosphere brightness on planetary disk when the ground albedo $A=0$, the visible distribution of intensity at different values of $\sigma$ (Figure 9) will be qualitatively similar to the observed intensity distribution found for the ultraviolet images of Saturn. However, the ratio of the reflectivities at $\mu=0.5$ and $\mu=1.0$ from the theoretical curve is about $1.4-1.6$ but the observed values are less than 1.1 (excluding the north component of equatorial belt where this ratio was about 1.17 in 1966). This discrepancy between the theory and observations can not be explained by the image quality, because the photographs of Saturn were obtained only in conditions of good seeing $(\sigma \leqslant 0.1)$. This means it is necessary to take into consideration the reflection of light from the ground (cloud layer of Saturn) in the ultraviolet, assuming $A \neq 0$. The observed limb brightening can not be explained by the outer scattering aerosol layer without also significant Rayleigh scattering in a gaseous layer.

Using the approximate formula

$$
r(\mu)=r_{a}(\mu) \exp \left(-\frac{2 \tau_{\mathrm{R}}}{\mu}\right)+r_{\mathrm{R}}\left(\mu, \tau_{\mathrm{R}}\right),
$$

where $r_{a}(\mu)=A \mu^{q}$ is the reflectivity (visible albedo) of the cloud cover, $r_{\mathrm{R}}$ is the reflectivity of the Rayleigh atmosphere, we can obtain the Rayleigh optical thickness $\tau_{\mathrm{R}}$ if $r(\mu)$ is known for different points of Saturn's disk. Passing by the details of the calculations (Teifel, 1974) we shall give here the final results. For Saturn's equatorial belt with a minimal reflectance in ultraviolet we have

$$
\tau_{\mathrm{R} 1} \approx 0.21 \pm 0.02,
$$

and the ground albedo

$$
A_{1} \approx 0.10 \pm 0.01 \text {. }
$$




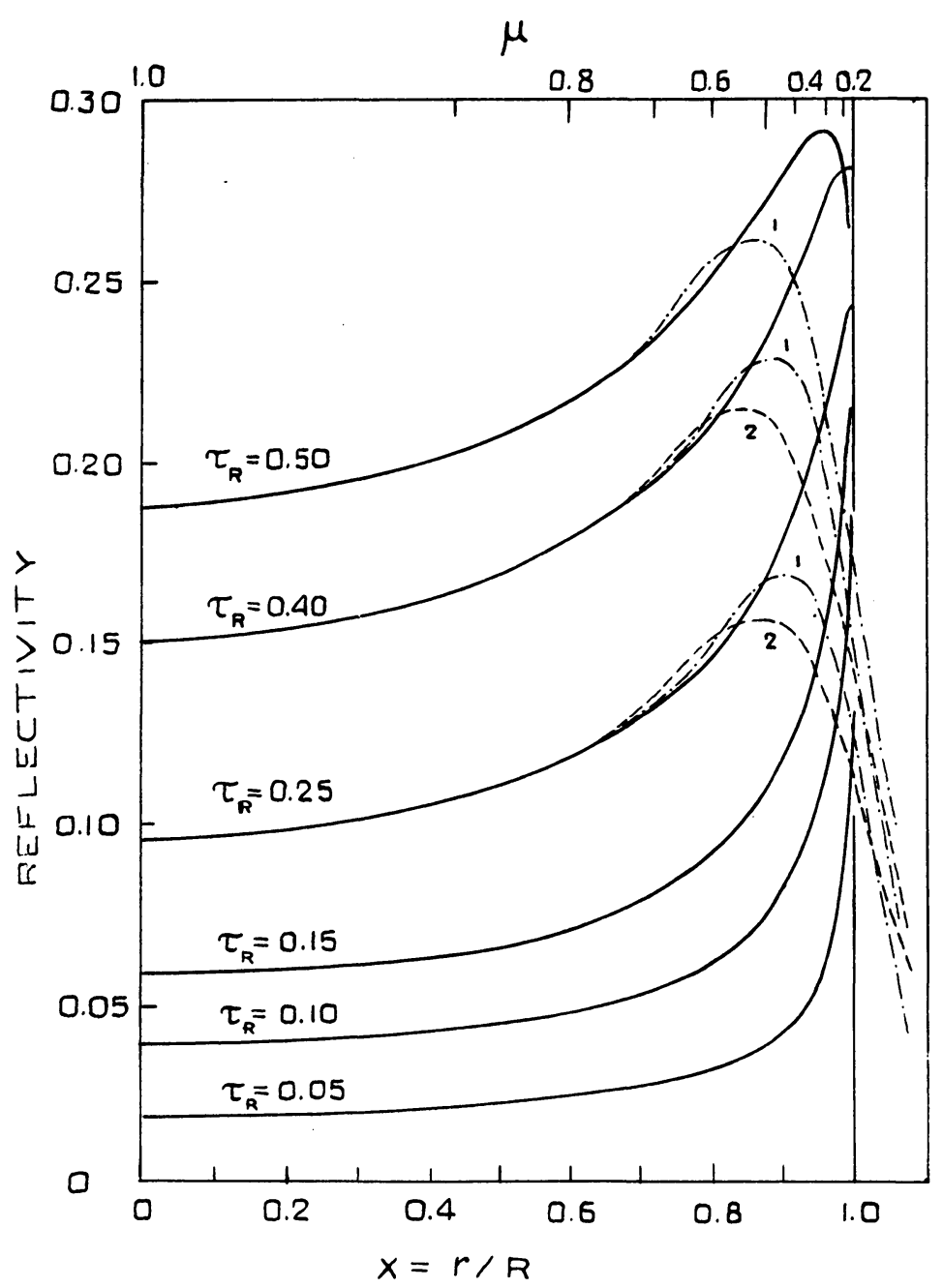

Fig. 9. Theoretical distribution of reflectivity for a Rayleigh atmosphere along the planetary radius, at some values of $\tau_{\mathrm{R}}$. Solid lines - without the atmospheric turbulence influence $(\sigma=0) ; 1-$ at $\sigma=0.050,2$ - at $\sigma=0.075$.

The maximal optical thickness of the Rayleigh atmosphere was obtained for the temperate belt of Saturn:

and

$$
\tau_{\mathrm{R} 2} \approx 0.30 \pm 0.03
$$

$$
A_{2} \approx 0.18 \pm 0.01
$$

As a Rayleigh scattering in Saturn's atmosphere is mostly caused by hydrogen (because the scattering coefficient of helium molecules is about 15 times less than that of hydrogen) it may be possible to estimate an equivalent thickness of $\mathrm{H}_{2}$ above the 
aerosol layer using the values of $\tau_{R}$ noted above. We have obtained

$$
\begin{aligned}
& U_{1}\left(\mathbf{H}_{2}\right) \approx 13.5 \pm 1.0 \mathrm{~km}-\mathrm{atm} \\
& U_{2}\left(\mathbf{H}_{2}\right) \approx 19.0 \pm 2.0 \mathrm{~km}-\mathrm{atm}
\end{aligned}
$$

The light exchange between the atmosphere and ground is neglected in formula (5). If the correction for this exchange would be introduced, we should have somewhat smaller values of $\tau_{\mathrm{R}}$ and $U\left(\mathrm{H}_{2}\right)$.

According to Trafton's (1967) calculations for a pure hydrogen atmospheric model of Saturn, the equivalent thickness of $\mathrm{H}_{2}$ above an upper boundary of the convective zone is about $50 \mathrm{~km}$-atm. For models calculated in the 'gray' approximation, with a pressure-induced absorption of the thermal radiation by hydrogen (Teifel, 1974), we have obtained about $30 \pm 2 \mathrm{~km}$-atm of $\mathrm{H}_{2}$ above the convective zone boundary. This value does not depend significantly on the assumed ratio $\mathrm{H}_{2}: \mathrm{He}$. Thus, from the ultraviolet observations we obtain smaller values of $U\left(\mathrm{H}_{2}\right)$ than would be observed if the upper boundary of the aerosol layer resembles the convective zone boundary. Probably the aerosol extends to higher levels (Figure 10), especially in the equatorial belt. As will be shown below, this conclusion also follows from the absorption bands measurements on Saturn's disk.

There is another peculiarity of the spectral reflectance of the equatorial and temperate belts on Saturn. As was shown by our photoelectric spectrophotometry, a

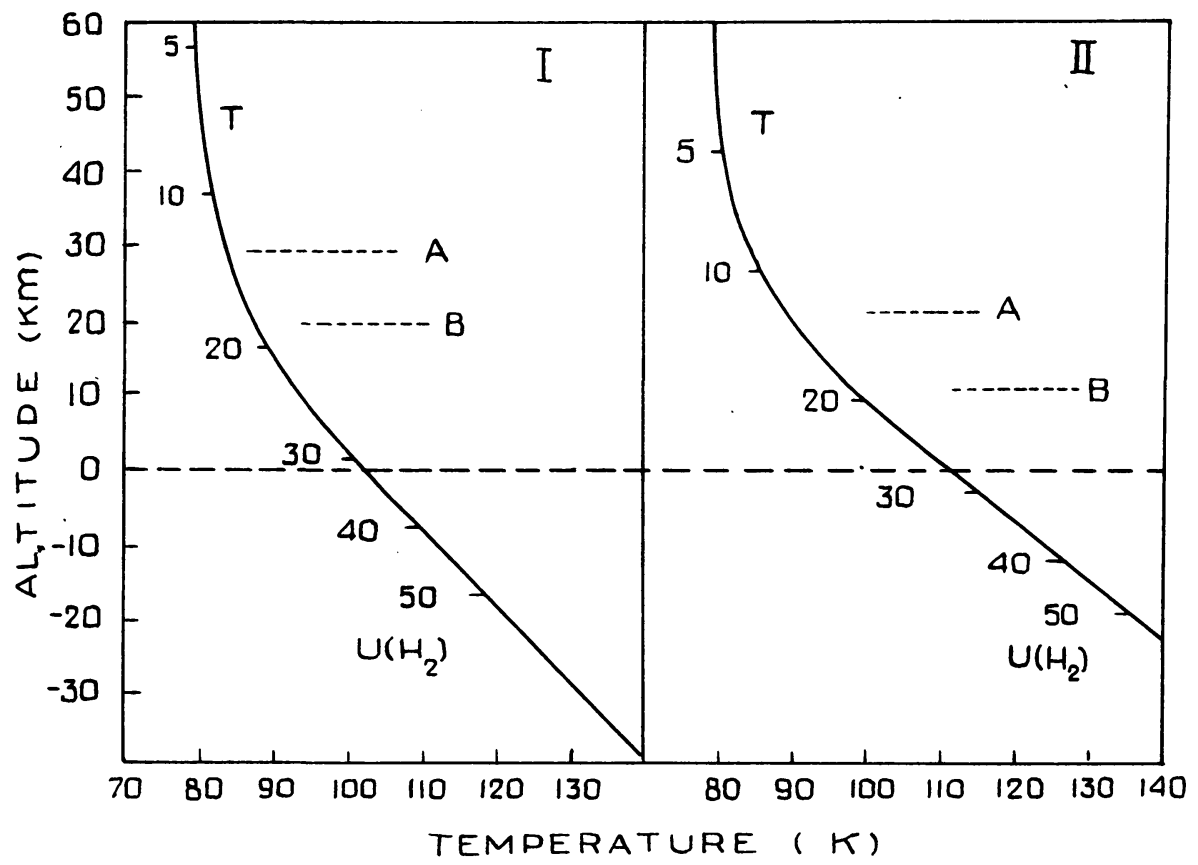

Fig. 10. The location of the upper boundary of the aerosol layer in the equatorial (A) and temperate (B) belts of Saturn, as derived from the ultraviolet measurements for two models of Saturn's atmosphere: $\mathrm{I}-\mathrm{H}_{2}: \mathrm{He}=5: 1, T_{e}=97 \mathrm{~K} ; \mathrm{II}-\mathrm{H}_{2}: \mathrm{He}=1: 1, T_{e}=97 \mathrm{~K}$. 


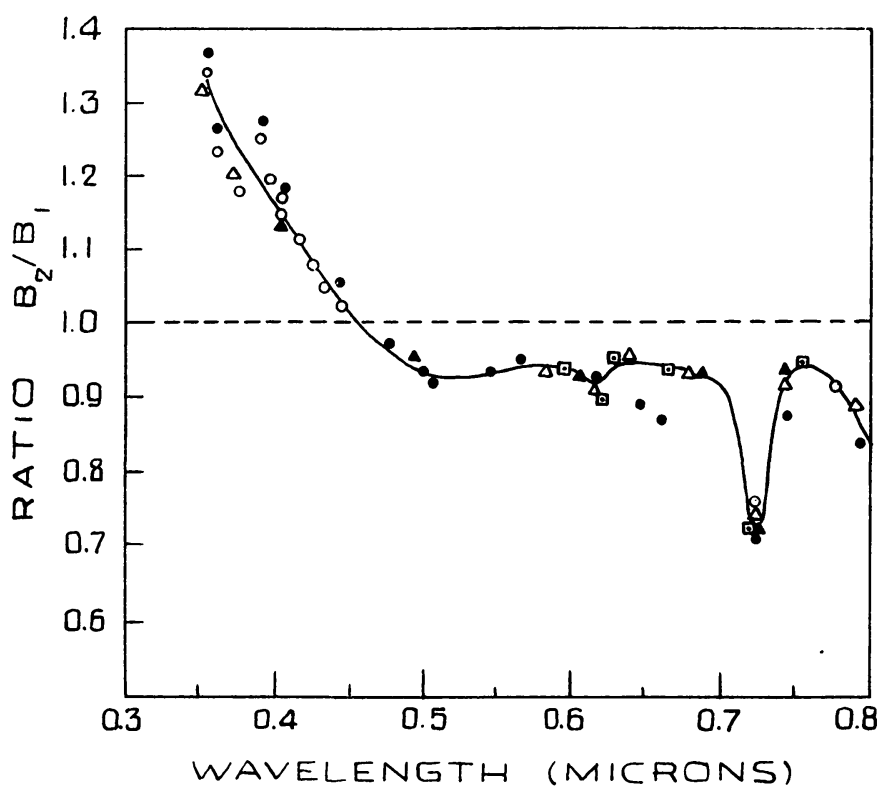

Fig. 11. The ratio of spectral reflectivities for the temperate $\left(B_{2}\right)$ and equatorial $\left(B_{1}\right)$ belts of Saturn in 1972, from the photoelectric spectral measurements.

sharp discrepancy in the spectral change of reflectivity between these belts begins shortward from $\lambda 0.5 \mu$. The ratio of the brightness of equatorial and temperate belts increases monotonically towards the longer wavelengths from $\lambda \approx 0.38 \mu$ to $\lambda 0.50 \mu$ (Figure 11); but at $\lambda>0.5 \mu$ the temperate belt appears more neutral as compared with the equatorial belt, excluding the methane absorption bands. The methane absorption in the temperate latitudes is more than in the equatorial belt, as can be seen from a comparison of spectral reflectivity within these bands. This is supported by measurements of the intensity of the methane bands.

An atmosphere above the clouds has produced some measurable effects at the longwavelengths only in the methane bands, but the reflectivity of Saturn in the continuum is specified by the cloud cover of planet. There are very few reliable data on the brightness distribution over Saturn's disk, since the photometry of the disk is more difficult when the saturnocentric latitude of the Earth is not equal to zero, because variations of cloud reflectivity have a zonal character.

We can estimate approximately the parameters for a scattering function of Saturn's cloud layer, using the data of the photographic photometry done in 1966 (Marin, 1968; Texereau, 1967) and the theoretical calculations of the brightness distribution for the scattering function

$$
X(\cos \gamma)=1+X_{1} P_{1}(\cos \gamma)+X_{2} P_{2}(\cos \gamma),
$$

where $P_{1}(\cos \gamma)$ and $P_{2}(\cos \gamma)$ are the Legendre polynomials (Loskutov, 1971). The best fit of the observations and theory (with the addition of the absolute spectrophoto- 


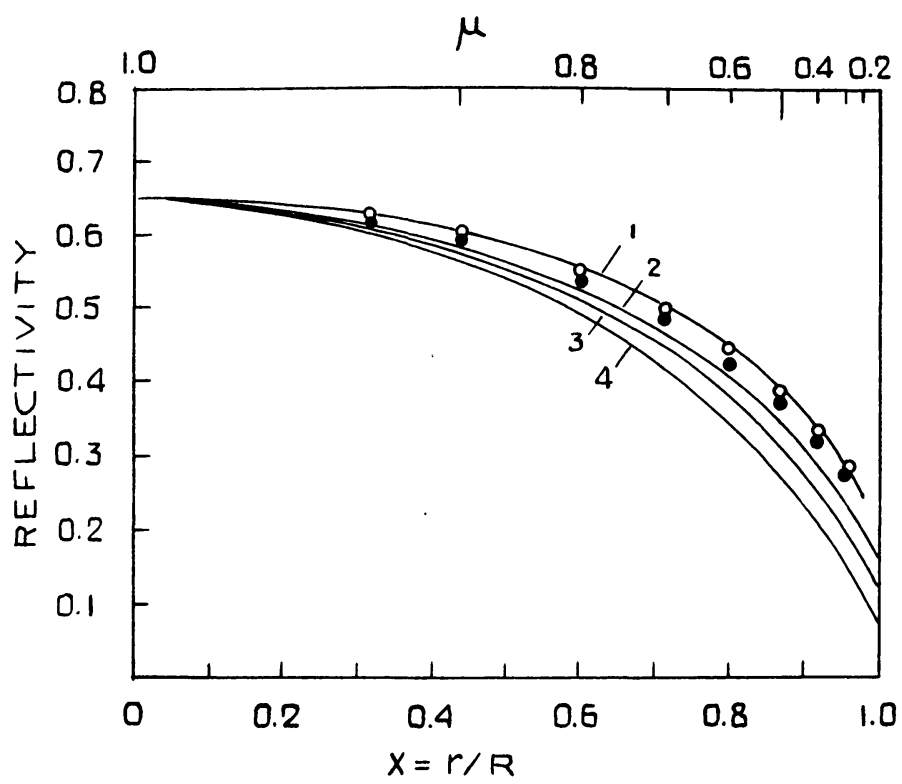

Fig. 12. The distribution of the reflectivity along the equatorial radius of Saturn's disk observed in yellow light and computed theoretically. Open circles - the observations of Texereau (1967), filled circles - the observations of Marin (1968). The parameters of the scattering function are $X_{2}=1.0$ and $X_{1}=1.0(1), 1.3(2), 1.5(3)$ and $1.7(4)$.

metry data) was obtained for the measurements of Saturn's images in yellow light $(\lambda \approx 0.59 \mu)$, if the parameters in (6) are $X_{1} \approx 1.10-1.15$ and $X_{2} \approx 1.0$ (Figure 12). Some discrepancies between the data of Marin and Texereau, obtained at the same time, may be explained by a not very exact determination of the limb on photographs. At any case, the observed darkening toward the limb of Saturn in yellow light is less than calculated for a scattering function with a great asymmetry. The relation of the parameter $\beta=B(\mu=0.5) / B(\mu=1.0)$, for the equator of Saturn in 1966 , with the reflectivity of the disk center at different wavelengths is very close to the theoretically calculated relation, as can be seen from Figure 13 for the case of $X_{1} \approx 1.1$ and $X_{2} \approx 1.0$. Nearly the same result was obtained by Krugov (1973) from measurements in red light. The scattering function for the Jovian clouds in the longwavelength region of the spectrum $(\lambda \approx 0.6 \mu)$, is more stretch forward $\left(X_{1} \approx 1.7, X_{2} \approx 1.0\right)$ as was obtained by Loskutov (1971) and Kartashov (1972).

Some results of Saturn's polarimetry were obtained recently (Hall and Riley, 1969; Bugaenko et al., 1971; Bugaenko and Galkin, 1972). These observations have not been interpreted, but it is interesting to note one strange result: the plane of polarization, near the west and east limbs, does not coincide with the reflection plane, either at zero phase angle or at another angle. The region of this anomalous behaviour of the polarization plane falls into the planetocentric latitudes $\pm 30^{\circ}$. This fact is noteworthy since the equatorial belt of Saturn is distinguished by its other optical properties. 


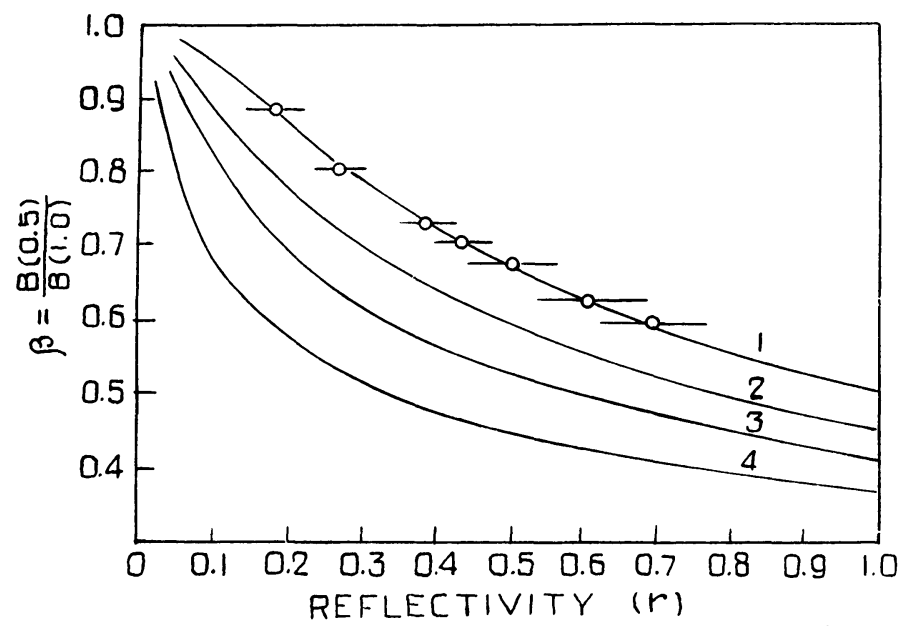

Fig. 13. The value of $\beta=\mathrm{B}(0.5) / \mathrm{B}(1.0)$ vs the spectral reflectivity $r$, from Marin observations of Saturn (dots), and theoretically computed for the parameters of the scattering function $X_{2}=1.0$ and $X_{1}=1.0(1), 1.3(2), 1.5(3)$ and $1.7(4)$.

\section{Morphology of the Methane Absorption on Disk of Saturn}

The first measurements of the intensity of the methane absorption band at $\lambda 0.62 \mu$ were carried out by Hess (1953) in 1950 . He found a relation between the increase of absorption and increasing latitude. More detailed studies of latitudinal variations of the intensity of this band on Saturn's disk were carried out in 1965-1970 in AlmaAta (Teifel, 1969; Teifel and Kharitonova, 1970; Teifel et al., 1971, 1973). As seen from the Figure 14, the least $\mathrm{CH}_{4}$ absorption was at temperate latitudes. It is important to note that the latitudinal distribution of this absorption is not changed significantly from year to year, independently of the inclination of Saturn's visible equator, which changed from $+3.5^{\circ}$ to $-22.5^{\circ}$ during this observational period. The last authors have shown that latitudinal variations in the absorption can not be stipulated by geometrical effects or by variations in planetary heating by solar radiation. The latitudinal variations in the methane absorption have reflected substantially the more or less permanent zonal features of planetary cloud cover. Kozyrev (1968) has also noted the attenuated absorption of $\mathrm{CH}_{4}$ in the equatorial belt, but he has attributed this effect to the region which fell inside of the ring shadow. However, the attenuation of methane absorption is a property of a wider equatorial belt and it is improbable to assume that the shadowing of Saturn's globe by the rings is the main reason for the decreasing of absorption near the equator.

The absorption in the $0.62 \mu$ band of $\mathrm{CH}_{4}$ is somewhat decreased from the center of disk toward the east and west limbs of Saturn as can be seen from Table II. This is in contradiction with the simple reflecting model for the formation of absorption bands. If this model is assumed the absorption band intensity must increase toward the limbs and the relation between the central depths of band at $\mu=1\left(R_{1}\right)$ and 

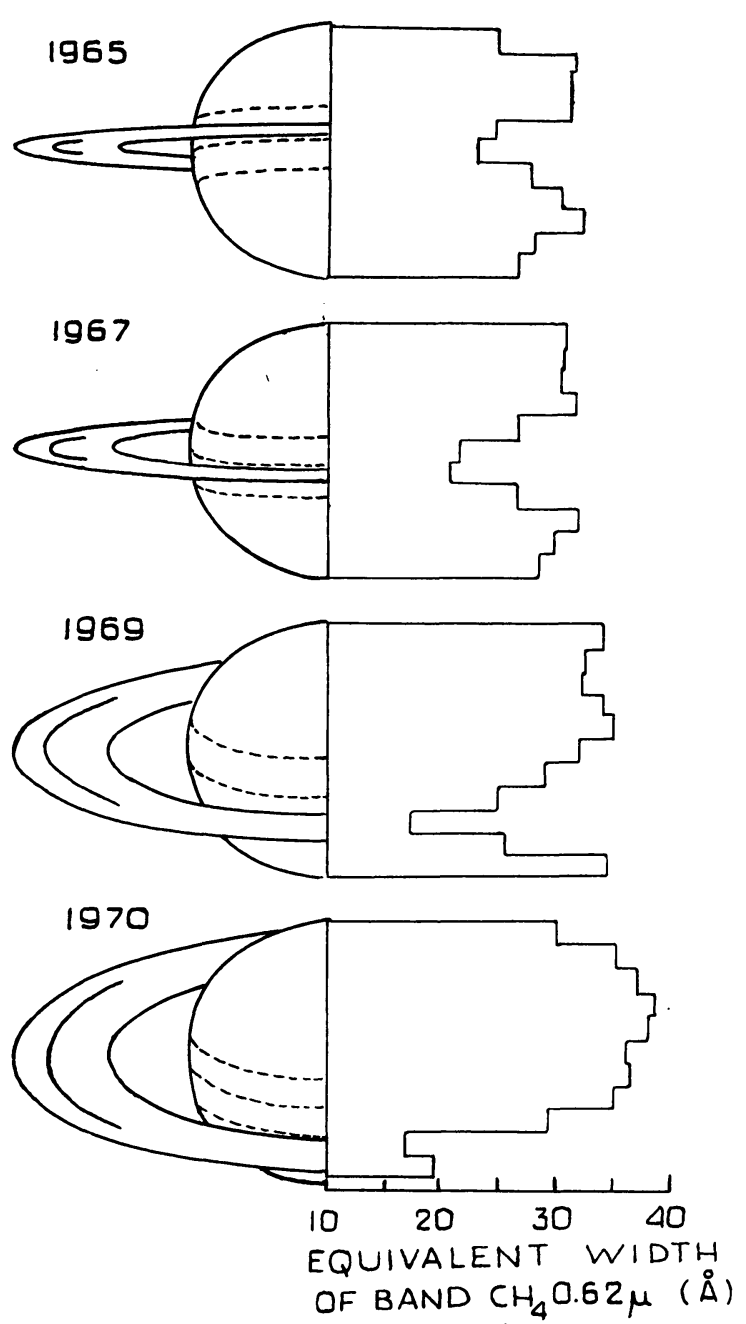

Fig. 14. The meridional distribution of methane absorption on Saturn in 1965-1970.

\section{TABLE II}

Equivalent width $(W)$ and central depth $(R)$ of the absorption band of $\mathrm{CH}_{4}$ at $0.62 \mu$ near the center $(\mu \approx 1)$ and near the limb $(\mu \approx 0.5)$ of Saturn's disk in 1969.

\begin{tabular}{rccccc} 
Latitudes & $\mu \approx 1.0$ & \multicolumn{3}{c}{$\mu \approx 0.5$} \\
& $W_{1}(\AA)$ & $R_{1}$ & $W_{2}(\AA)$ & $R_{2}$ & $R^{*}{ }^{2}$ \\
& & & & & \\
$0^{\circ}-14$ & 29.2 & 0.276 & 26.5 & 0.255 & 0.480 \\
$-14-28$ & 32.9 & 0.316 & 32.8 & 0.315 & 0.525 \\
$-28-45$ & 34.5 & 0.346 & 33.8 & 0.336 & 0.572
\end{tabular}


$\mu=0.5\left(R_{2}\right)$ may be presented as a simple formula,

$$
R_{2}=1-\left(1-R_{1}\right)^{2},
$$

since for the depth of a band consisting of many strongly overlapped lines

$$
R_{v}=1-\exp \left(-\frac{2 \tau_{v}}{\mu}\right),
$$

where $\tau_{v}$ is the optical thickness of the atmosphere above the clouds in the absorption band center, $\mu=\mu_{0}=\cos \varepsilon$.

The depth of a methane band near Saturn's limb would satisfy relation (8) if the simple reflecting model were true, and would be considerably more than the observed depth, as seen from the last column of Table II.

On the other hand if we assume a scattering model for the formation of the absorption bands, the depth of the band must satisfy the expression

$$
R_{v}=1-\frac{\varrho_{v}\left(\omega_{v}, \mu\right)}{\varrho_{c}\left(\omega_{c}, \mu\right)},
$$

where $\varrho_{c}$ and $\varrho_{v}$ are the reflectivities of the cloud layer in the continuum and in the absorption band center respectively. The relation between $R_{1}$ and $R_{2}$ (the band depths at $\mu=1$ and $\mu=0.5$ ) will be practically the same for any scattering function (Figure 15).

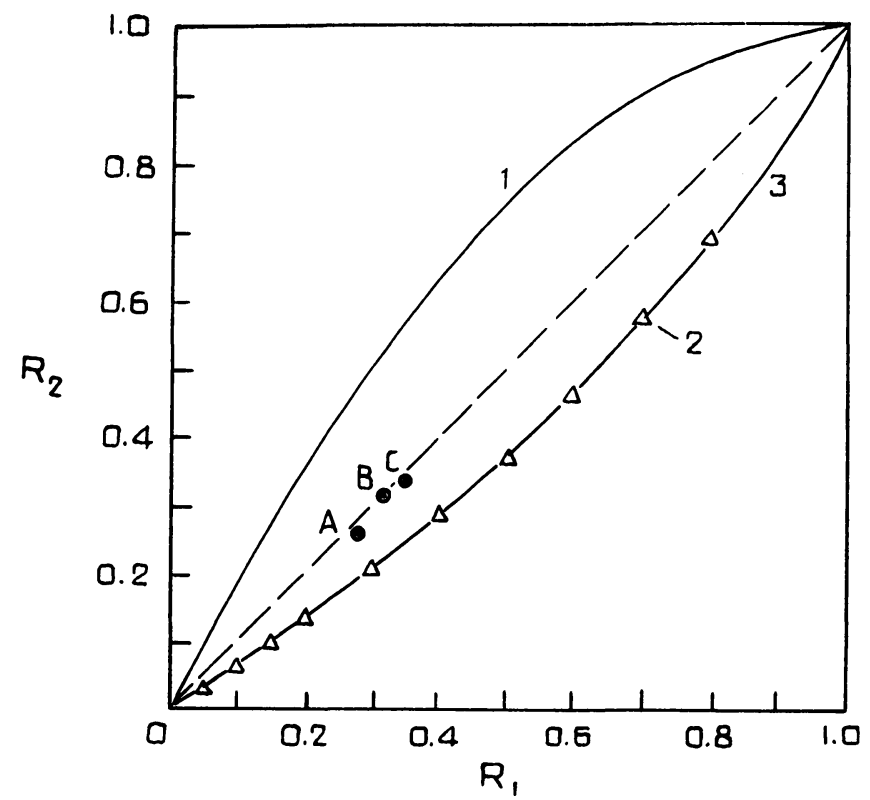

Fig. 15. Theoretically computed and observed relation between the depths of the $0.62 \mu \mathrm{CH}_{4}$ absorption band; $R_{1}(\mu \approx 1.0)$ and $\mathbf{R}_{2}(\mu \approx 0.5)$. A - for the equatorial belt, $\mathrm{B}$ - for the temperate belt, $\mathrm{C}$ - for the higher latitudes of Saturn. Curves: 1 - for the simple reflecting model, 2 and 3 - for the scattering models with isotropic scattering (2) and with the scattering function characterized by $X_{1}=1.7$ and $X_{2}=1.0$. 
The observations do not fit this relationship, as noted on Figure 15 by points. It may be interpreted as a result of the absorption in the overcloud atmosphere of Saturn at the sufficient role of the multiple scattering inside the clouds in the absorption band formation. From the comparison of the observations with the theory we have concluded that the optical thickness of the outer atmosphere in $0.62 \mu$ band of $\mathrm{CH}_{4}$ is the

\section{TABLE III}

Latitudinal variations of the methane absorption in the bands of $\mathrm{CH}_{4}$ at $0.62 \mu$ and $0.72 \mu$ on Saturn's disk from the photoelectric measurements in 1971

\begin{tabular}{|c|c|c|c|c|c|c|c|}
\hline \multirow[t]{2}{*}{ No } & \multirow[t]{2}{*}{ Latitudes } & \multicolumn{3}{|l|}{$\mathrm{CH}_{4} 0.62 \mu$} & \multicolumn{3}{|l|}{$\mathrm{CH}_{4} 0.72 \mu$} \\
\hline & & $R$ & $W(\AA)$ & $n$ & $R$ & $W(\AA)$ & $n$ \\
\hline 1 & $+13^{\circ}--3^{\circ}$ & $0.238 \pm 0.003$ & $25.7 \pm 0.4$ & 88 & $0.600 \pm 0.004$ & $110.7 \pm 1.2$ & 90 \\
\hline 2 & $+1--14$ & $0.263 \pm 0.002$ & $27.9 \pm 0.4$ & 62 & $0.666 \pm 0.003$ & $122.5 \pm 1.2$ & 52 \\
\hline 3 & $-10--24$ & $0.287 \pm 0.002$ & $29.6 \pm 0.4$ & 65 & $0.712 \pm 0.003$ & $132.1 \pm 0.9$ & 52 \\
\hline 4 & $-20--35$ & $0.295 \pm 0.002$ & $31.4 \pm 0.3$ & 111 & $0.760 \pm 0.002$ & $138.7 \pm 0.9$ & 87 \\
\hline 5 & $-31--46$ & $0.292 \pm 0.002$ & $29.8 \pm 0.4$ & 71 & $0.770 \pm 0.003$ & $141.0 \pm 1.4$ & 44 \\
\hline 6 & $-42--60$ & $0.282 \pm 0.002$ & $30.2 \pm 0.4$ & 68 & $0.755 \pm 0.002$ & $135.3 \pm 1.3$ & 59 \\
\hline 7 & $-55--77$ & $0.278 \pm 0.003$ & $29.6 \pm 0.6$ & 64 & $0.742 \pm 0.003$ & $136.6 \pm 1.7$ & 50 \\
\hline 8 & $-68--90$ & $0.288 \pm 0.004$ & $32.2 \pm 0.7$ & 46 & $0.735 \pm 0.003$ & $132.4 \pm 1.8$ & 45 \\
\hline
\end{tabular}

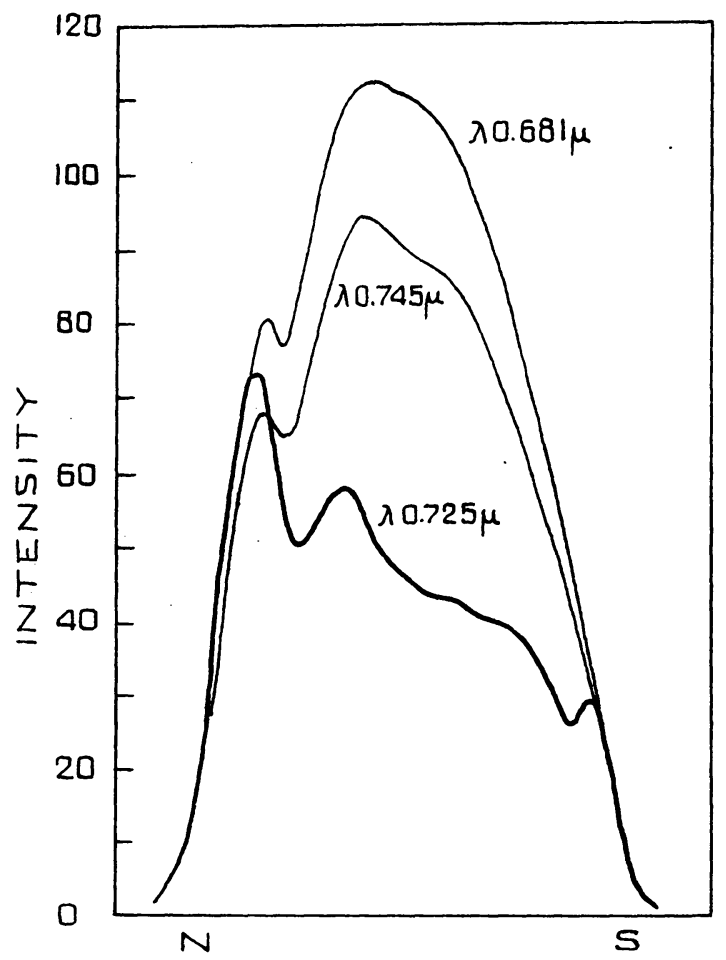

Fig. 16. The photoelectric monochromatic profiles of Saturn's central meridian in 1972, in the center of the $0.72 \mu \mathrm{CH}_{4}$ absorption band and in the nearest parts of the continuous spectrum. 
least in the equatorial belt of Saturn and is increased about 1.5 times in temperate belts of this planet. The volume scattering coefficient is varied very slightly with the latitude and the latitudinal discrepancy of the optical thickness of the outer atmosphere has suggested that the upper boundary of the cloud layer in the equatorial belt is located somewhat higher than in the temperate latitudes. The latitude difference is about $12-13 \mathrm{~km}$ at $T \approx 100 \mathrm{~K}$. Let us remember that the same conclusion was reached independently from the observations of Saturn in the ultraviolet.

In 1971 and 1972-1973 measurements of $\mathrm{CH}_{4}$ absorption bands at $\lambda 0.62 \mu$ and $0.72 \mu$ were carried out on Saturn's disk with a photoelectric spectrometer (Teifel and Kharitonova, 1972b, 1974). The latitudinal variations of the equivalent widths and central depths of these bands were studied and the results for 1971 are listed in Table III where $n$ is the number of the measured absorption band profiles.

The change of the methane absorption with latitude is noticeable even on the photometric scans of the central meridian of Saturn: the brightness distribution in the $\mathrm{CH}_{4} 0.72 \mu$ band is sharply different from the distribution in the nearest parts of continuum (Figure 16). A similar picture is observed in the more intense $\mathrm{CH}_{4}$ band $0.89 \mu$ as shown by photographs obtained by Owen (1969) and infrared spectrograms (Figure 17) obtained by Vdovitchenko (1974).

The results of several series of absorption bands measurements near the west and east limbs of Saturn in 1972 are shown in Figure 18. These data have confirmed the

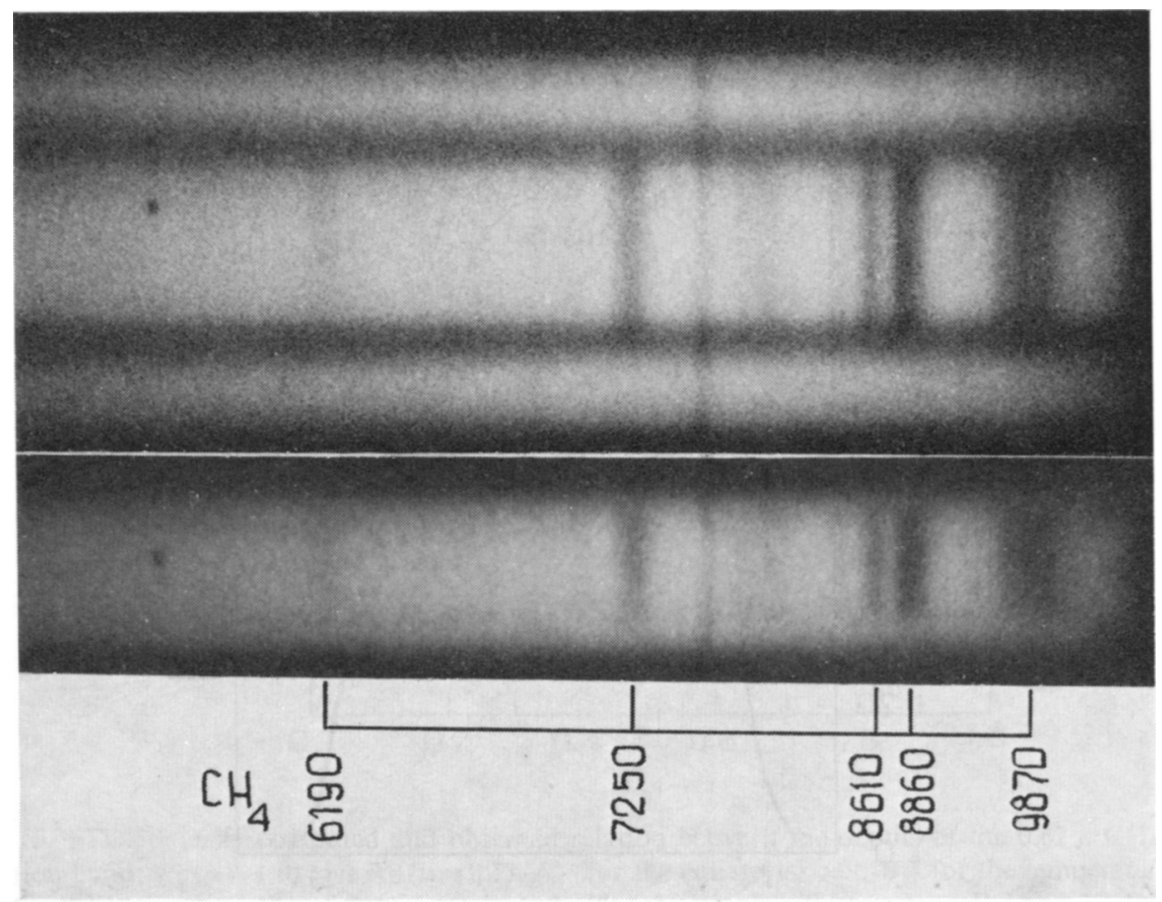

Fig. 17. The infrared spectrograms of Saturn's equator (1) and of the central meridian (2), at $\lambda \lambda 0.6-1.1 \mu$, photographed by Vdovitchenko in 1972 using the electronic image tube. 


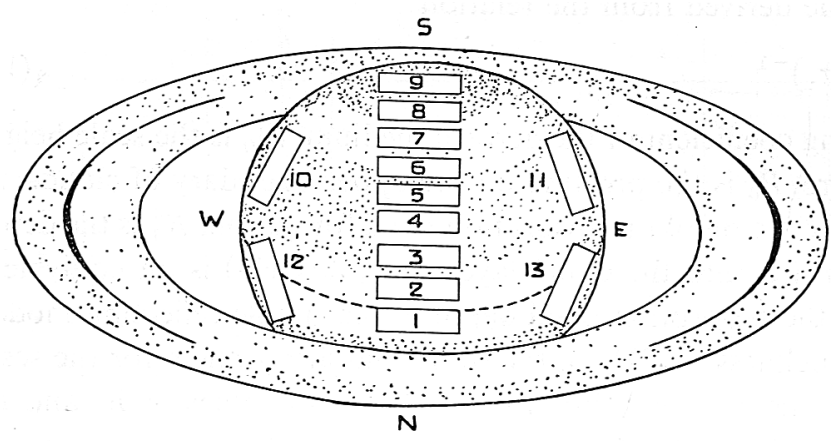

1972

\begin{tabular}{|c|c|c|}
\hline$N$ & $R \quad 0.62 \mu$ & $R 0.72 \mu$ \\
\hline 1 & $0.243 \pm 0.006$ & $0.632 \pm 0.006$ \\
2 & $0.258 \pm 0.004$ & \\
3 & $0.280 \pm 0.004$ & $0.733 \pm 0.008$ \\
4 & $0.289 \pm 0.003$ & $0.763 \pm 0.005$ \\
5 & $0.287 \pm 0.003$ & $0.776 \pm 0.008$ \\
6 & $0.269 \pm 0.004$ & \\
7 & $0.258 \pm 0.005$ & $0.706 \pm 0.008$ \\
8 & $0.254 \pm 0.007$ & \\
9 & $0.265 \pm 0.008$ & $0.689 \pm 0.006$ \\
\hline 10 & $0.282 \pm 0.006$ & $0.747 \pm 0.009$ \\
11 & $0.293 \pm 0.004$ & $0.769 \pm 0.005$ \\
12 & $0.236 \pm 0.004$ & $0.682 \pm 0.006$ \\
13 & $0.240 \pm 0.007$ & $0.672 \pm 0.007$ \\
\hline
\end{tabular}

Fig. 18. Estimates of the central depth of the methane absorption bands at $0.62 \mu$ and $0.72 \mu$, in some parts of Saturn's disk, obtained from the photoelectric spectral measurements in 1972.

conclusion about the lack of increasing absorption toward the limbs within each belt of Saturn.

An interesting result was obtained both in 1971 and 1972. The intensity of the $\mathrm{CH}_{4}$ absorption band at $0.62 \mu$ is slightly increased near the south polar limb as compared with the adjoining regions of disk but the $\mathrm{CH}_{4}$ band at $0.72 \mu$ does not show this effect.

From the measurements of the intensity of the $\mathrm{CH}_{4}$ band at $0.62 \mu$ in 1971 we have obtained some characteristics of the cloud layer and the atmosphere above the clouds. These are presented in Table IV where $\varrho_{c}$ are the reflectivities in the continuum of zones near the central meridian $(\lambda \approx 0.62 \mu), R_{1}$ and $R_{2}$ are the observed values of the absorption band depth at $\mu \approx 1.0$ and $\mu \approx 0.5, \tau_{v}^{*}$ is the optical thickness of the atmosphere above the clouds in the center of the absorption band for the simple re-

TABLE IV

Some atmospheric parameters derived from the $\mathrm{CH}_{4} 0.62 \mu$ absorption band measurements on Saturn's disk in 1971

\begin{tabular}{llllllll}
\hline Parameter & \multicolumn{2}{l}{ Equatorial belt } & \multicolumn{3}{l}{ Temperature belt } \\
\cline { 2 - 4 } & I & II & III & I & II & III \\
\hline$Q_{c}$ & 0.775 & 0.775 & 0.775 & 0.736 & 0.736 & 0.736 \\
$R_{1}$ & 0.275 & 0.288 & 0.262 & 0.297 & 0.314 & 0.290 \\
$R_{2}$ & 0.252 & 0.239 & 0.265 & 0.316 & 0.299 & 0.333 \\
$\tau^{*}{ }_{v}$ & 0.161 & 0.170 & 0.152 & 0.177 & 0.189 & 0.172 \\
$\tau_{v}{ }_{v} P_{o}$ & 0.030 & 0.019 & 0.040 & 0.055 & 0.044 & 0.067 \\
$\sigma_{\mathrm{a}} H_{0}{ }_{P_{e}}$ & 2.5 & 1.3 & 4.1 & 5.0 & 3.5 & 7.7 \\
$R^{*}{ }_{1}$ & 0.230 & 0.261 & 0.200 & 0.215 & 0.240 & 0.189 \\
$L\left(\mathrm{CH}_{4}\right)$, m-atm & 218 & 230 & 205 & 239 & 255 & 232 \\
$U\left(\mathrm{CH}_{4}\right)$, m-atm & 40 & 26 & 54 & 74 & 59 & 90
\end{tabular}


flecting model, $\tau_{v}$ is the same but for a model with a multiple aerosol scattering, $b^{-1}=\sigma_{a} H_{0}\left(\mathrm{P}_{0} / \mathrm{P}_{e}\right)$ is a value derived from the relation

$$
\omega_{v}=\left(\omega_{c}^{-1}+b \tau_{v}\right)^{-1}
$$

and $\sigma_{a}$ is a volume scattering coefficient of the aerosol medium, $H_{0}$ is the scale height of the overcloud atmosphere, $P_{0}$ is the pressure on the upper boundary of clouds, $P_{e}$ is the effective pressure in the region of the absorption band formation, $R_{1}^{*}$ is the depth of the absorption band formed into the cloud layer only, $L\left(\mathrm{CH}_{4}\right)$ is an equivalent path of $\mathrm{CH}_{4}$ derived from the absorption band depth in the simple reflecting model, $U\left(\mathrm{CH}_{4}\right)$ is an equivalent thickness of $\mathrm{CH}_{4}$ in the overcloud atmosphere for the scattering model. All these parameters were obtained for the mean values of $R_{1}$ and $R_{2}$ (the column index I) and for the probable limits of $R_{1}$ and $R_{2}$ in the confidence interval at the probability $\alpha=0.99$ (indices II and III).

As means we have $U\left(\mathrm{CH}_{4}\right) \approx 41 \mathrm{~m}$-atm in the equatorial belt and $U\left(\mathrm{CH}_{4}\right) \approx 72 \mathrm{~m}$ atm in the temperate belt with the probable error $\pm 10 \mathrm{~m}$-atm. The upper limit of the $\mathrm{CH}_{4}$ relative abundance is about $3.8 \times 10^{-3}$ if we assume that the thickness of the overcloud atmosphere is the same in both the ultraviolet and visible spectral range.

Assuming $H_{0} \approx 30 \mathrm{~km}$ and $P_{e} / P_{0} \approx 2$ we obtain $\sigma_{a} \approx(1-3) \times 10^{-6} \mathrm{~cm}^{-1}$ in the equatorial belt and $\sigma_{a} \approx(2-5) \times 10^{-6} \mathrm{~cm}^{-1}$ in the temperate belt.

\section{Some Notes on the Saturn's Atmosphere Structure}

Let us consider to what extent the observational data agree with a probable atmospheric model for Saturn. We assume as a working model the 'gray' one with $T_{e}=97 \mathrm{~K}$ and the ratio $\mathrm{H}_{2}: \mathrm{He}=5: 1$ for the number of molecules. The altitudinal temperature and pressure distributions in this model are presented on Figure 19, where curves of the changes of saturated $\mathrm{NH}_{3}$ and $\mathrm{CH}_{4}$ vapour pressures are also shown. The imimpossibility of methane condensation at the Saturn's atmospheric conditions is evident because the relation

$$
P_{\mathrm{CH}_{4}}(z)>E_{\mathrm{CH}_{4}}\left(T_{z}\right)
$$

is necessary for the formation of the $\mathrm{CH}_{4}$ clouds. Here $\boldsymbol{P}_{\mathrm{CH}_{4}}(z)$ is the partial pressure of $\mathrm{CH}_{4}$ at the level $z$ and $E_{\mathrm{CH}_{4}}\left(T_{z}\right)$ is the pressure of saturated vapours of $\mathrm{CH}_{4}$ at the temperature of this level. As seen from Figure 19 the partial pressure of $\mathrm{CH}_{4}$ must be no less than 0.2 of the general pressure for the presence of the methane clouds. In this case, the relative abundance of $\mathrm{CH}_{4}$ must be at least about $3 \times 10^{-2}$ of the molecules, but the observations give us a value which is about ten times smaller.

For the ammonia condensation the conditions in the atmosphere of Saturn are more favourable since the pressure of saturated vapours of $\mathrm{NH}_{3}$ is sharply decreased with the temperature decreasing upward. If the relative abundance of $\mathrm{NH}_{3}$ is assumed about $4 \times 10^{-5}$ in the undercloud atmosphere, based on the radio observations, the partial pressure of ammonia under the clouds must be about $3 \times 10^{-4} P$ where $P$ is a general pressure. If so, the sublimation level (or lower boundary of the ammonia 


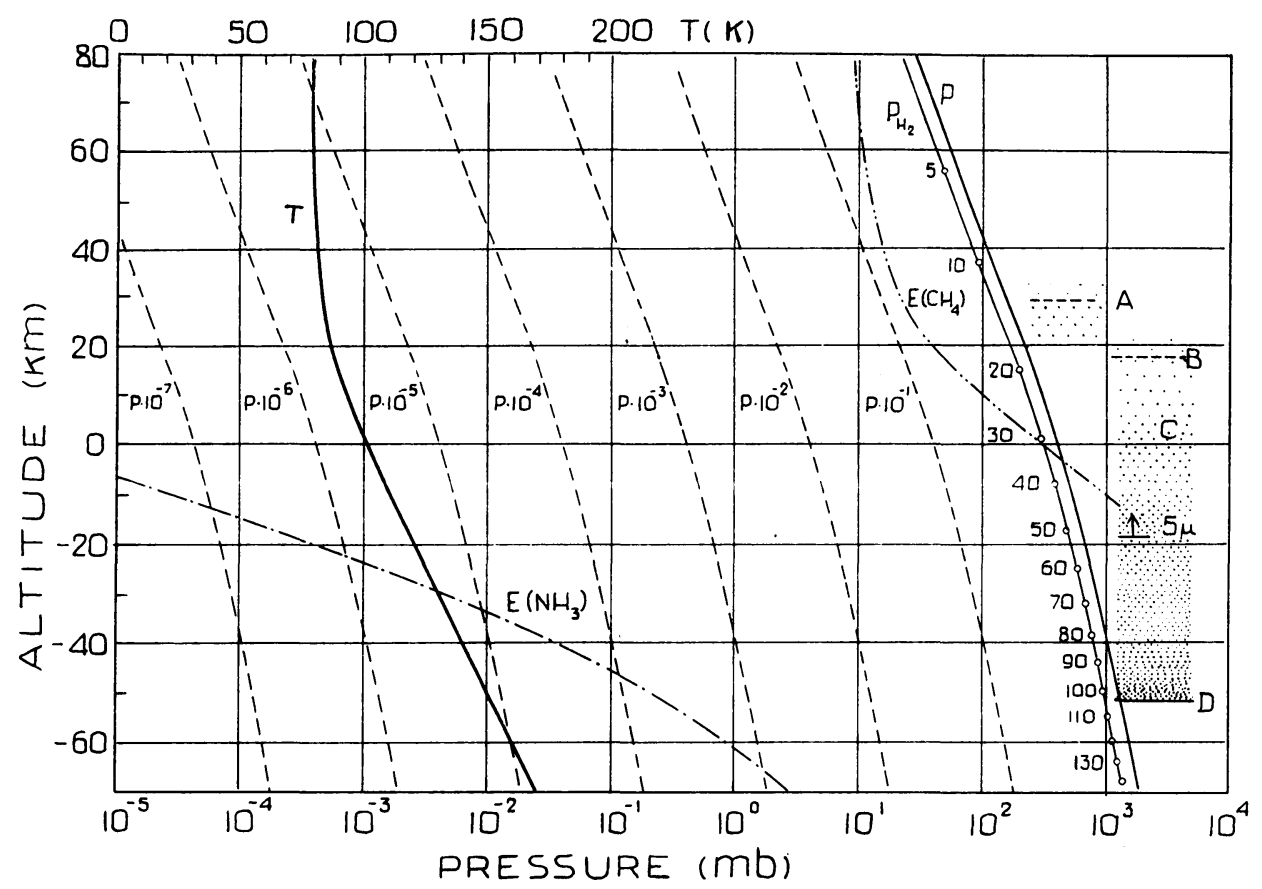

Fig. 19. The working model of the atmosphere of Saturn, A - the upper boundary of the aerosol in the equatorial belt, $\mathrm{B}$ - the same in the temperate belt, $\mathrm{C}$ - the level of the theoretical boundary of the convective zone, D - the $\mathrm{NH}_{3}$ sublimation level. For this model $T_{e}=97 \mathrm{~K}, \mathrm{H}_{2}: \mathrm{He}=5: 1$.

clouds) must be at a depth of about $55 \mathrm{~km}$ below the convective zone boundary. The temperature of this level is about $155 \mathrm{~K}$. The probable range on the depth of the lower boundary for ammonia clouds is from 50 to $60 \mathrm{~km}$, if some uncertainty in the relative abundance of $\mathrm{NH}_{3}$ is taken into consideration.

Thus the vertical extent of the aerosol layer on Saturn is very significant: using the ultraviolet data, it is about $70-80 \mathrm{~km}$, i.e. about 2-3 times more than the probable thickness of the upper cloud layer on Jupiter. The lower part of the cloud cover of Saturn is rather dense and opaque, even for infrared radiation (at least for $\lambda \leqslant 5 \mu$ ). As was noted above, the brightness temperature of Saturn at $\lambda 5 \mu$ is about $120 \mathrm{~K}$. This temperature may be connected with a level which is about $30-40 \mathrm{~km}$ above the lower boundary of the ammonia clouds.

Theoretical computations (Teifel, 1970; Teifel et al., 1971) show that the density of the ammonia cloud layer in the convective zone must be comparable with the density of Earth stratus clouds (about $10^{-7} \mathrm{~g} \mathrm{~cm}^{-3}$ ). Formation of clouds is also feasible over the upper boundary of the convective zone, because even a very small quantity of $\mathrm{NH}_{3}$ transported to these layers by mechanical turbulence may produce the oversaturation. The ammonia sublimation process may be facilitated at these small concentrations by the presence of cosmic dust in the upper atmosphere, and by the products of meteor particle evaporation. The density of the aerosol component at these 
latitudes depends on the vertical mixing intensity, and on the number of particles having a meteor nature which play the role of sublimation nuclei. The aerosol component in the upper cloud zone is a comparatively thin haze, and the particles of this haze may be of a two-layer structure - a solid nucleous with an ice envelope. The optical properties of the haze depend on the particle structure. The problem of the cloud colouring is not solved for Saturn, as for Jupiter. Some chemical compounds as $\mathrm{NH}_{4} \mathrm{SH}$ or $\left(\mathrm{NH}_{4}\right)_{2} \mathrm{~S}$ whose presence in Saturn's atmosphere is supposed by Lewis (1969), Owen and Mason (1969) and Lebofsky (1973) do not contradict the spectral reflectivity of Saturn, but the formation of these substances seems more probable in the deeper layers of the atmosphere.

The cloud cover of Saturn is the only indicator of the atmosphere circulation. Some unstability of the atmosphere is detected from time to time. Thus, for instance in 1966 the south component of Saturn's equatorial belt was a light zone both in visible light and ultraviolet, and the brightness distribution in this zone was significantly different from the distribution in other cloud belts of Saturn (as seen from Figure 6). In 1968 this anomalous component had disappeared. Another interesting feature on Saturn was observed in 1970 (Reese, 1970). It was a little light spot at a latitude of about $-57^{\circ}$.

The spectral reflectivity differences between the equatorial and temperate belts at $\lambda<0.5 \mu$ can not be explained only by differences in the overcloud Rayleigh atmospheric thickness. There is the possibility of an increase in the concentration of a strongly absorbing substance in the equatorial belt. This substance may be formed there at the higher levels.

A few words concerning the great equatorial acceleration on Saturn may be added. It is known that the rotation periods are about $10^{\mathrm{h}} 14^{\mathrm{m}}$ at the equator and about $10^{\mathrm{h}} 38^{\mathrm{m}}$ at latitudes higher than $30^{\circ}$. If this difference of zonal rotational velocities is attributed to the same equipotential level in the atmosphere, the differential velocity must be more than $330 \mathrm{~m} \mathrm{~s}^{-1}$ (this velocity on Jupiter is about $120 \mathrm{~m} \mathrm{~s}^{-1}$ ). At the boundary between the equatorial and temperate belts of Saturn, there is no turbulence which is so strong as that on Jupiter. On the other hand, we do not detect on Jupiter a significant difference between the intensity of molecular absorption bands in zones with rotation systems I and II. Apparently, the altitude differences of the cloud boundary on Jupiter are less than on Saturn. The visible equatorial acceleration on Saturn, and the higher location of the aerosol boundary are probably connected with one another. Two alternative assumptions may be offered. (1) The equatorial acceleration on Saturn is truly strong and the formation of clouds at higher levels on the equator is promoted by the presence of this acceleration; (2) The true equatorial acceleration is not as strong as it appears but the high clouds on equator are moved more quickly by the stratospheric winds. On both cases, the observed peculiarities of Saturn's atmospheric circulation are more probably caused by inner factors than by solar heating input.

\section{References}

Allen, D. A. and Murdock, T. L.: 1971, Icarus 14, 1.

Aumann, H. H., Gillespie, C. M., and Low, F. J.: 1969, Astrophys. J. 157, 69. 
Berge, C. L. and Read, R. B.: 1968, Astrophys. J. 152, 755.

Bugaenko, L. A.: 1972, Astron. Vestnik 6, 19 (in Russian).

Bugaenko, L. A., Galkin, L. S., and Morozhenko, A. V.: 1971, Astron. Zh. 48, 602 (in Russian).

Bugaenko, O. I. and Galkin L. S.: 1972, Astron. Zh. 49, 837 (in Russian).

Coulson, K., Dave, J. V., and Sekera, Zd.: 1960, Tables Related to Radiation Emerging from a Planetary Atmosphere with Rayleigh Scattering, Univ. of Calif. Press.

Fink, U. and Belton, M. J. S.: 1969, J. Atmospheric Sci. 26, 952.

Giver, L. P. and Spinrad, H.: 1966, Icarus 5, 586.

Hall, J. S. and Riley, L. A.: 1969, J. Atmospheric Sci. 26, 920.

Hess, S. L.: 1953, Astrophys. J. 118, 151.

Irvine, W. M. and Lane, A. P.: 1971, Icarus 15, 18.

Kartashov, V. F.: 1972, Astron. Tzirkular, No. 724 (in Russian).

Keay, C. S. L., Low, F. J., and Rieke, G. H.: 1972, Sky Telesc. 44, 296.

Kellermann, K. I.: 1966, Icarus 5, 478.

Kozyrev, N. A.: 1968, Izv. GAO Akad. Nauk SSSR, No. 184, 99 (in Russian).

Krugov, V. D.: 1972, Astron. Vestnik 6, 85 (in Russian).

Krugov, V. D.: 1973, Dissertation (in Russian).

Kuiper, G. P.: 1947, The Atmospheres of the Earth and Planets, Chicago, p. 304.

Kuzmin, A. D., Naumov, A. P., and Smirnova, T. V.: 1972, Astron. Vestnik 6, 13 (in Russian).

Lebofsky, L. A.: 1973, Bull. Am. Astron. Soc. 4, 362.

Lewis, J. S.: 1969, Icarus 10, 365.

Loskutov, V. M.: 1971, Astron. Vestnik 5, 153 (in Russian).

Low, F. J.: 1964, Astron. J. 69, 550.

Low, F. J. and Davidson, A. W.: 1969, Bull. Am. Astron. Soc. 1, 200.

Marin, M.: 1968, J. Obs. 51, 179.

McCord, T. B., Johnson, T. V., and Elias, J. H.: 1971, Astrophys. J. $165,413$.

Menzel, D., Coblentz, W., and Lampland, C.: 1926, Astrophys. J. 63, 177.

Moroz, V. I.: 1966, Astron. Zh. 38, 1080 (in Russian).

Moroz, V. I., Vasiltchenko, N. V., Danilanz, L. B., and Kaufman, S. A.: 1968, Astron. Zh. 45, 189 (in Russian).

Murphy, R. E.: 1973, preprint.

Murray, B. C. and Wildey, R. L.: 1963, Astrophys. J. 137, 692.

Owen, T.: 1969, Icarus 10, 355.

Owen, T. and Mason, H. P.: 1969, J. Atmospheric Sci. 26, 870.

Reese, E. J.: 1971, Icarus 15, 466.

Schoenberg, E.: 1929, Handbuch der Astrophysik, Band II, Berlin.

Spinrad, H.: 1964, Appl. Opt. 3, 181.

Tanaka, W.: 1967, Proc. Japan. Acad. 43, 971.

Teifel, V. G.: 1969, J. Atmospheric Sci. 26, 854.

Teifel, V. G.: 1970, Astron. Vestnik 4, 81 (in Russian).

Teifel, V. G.: 1974, Astron. Vestnik 8, 3 (in Russian).

Teifel, V. G., Usoltzeva, L. A., and Kharitonova, G. A.: 1971, in C. Sagan, T. C. Owen, and H. J. Smith (eds.), 'Planetary Atmospheres', IAU Symp. 40, 375.

Teifel, V. G., Usoltzeva, L. A., and Kharitonova, G. A.: 1971, Astron. Zh. 48, 380 (in Russian).

Teifel, V. G., Usoltzeva, L. A., and Kharitonova, G. A.: 1973, Astron. Zh. 50, 167 (in Russian).

Teifel, V. G. and Kharitonova, G. A.: 1970, Astron. Tzirkular, No. 549 (in Russian).

Teifel, V. G. and Kharitonova, G. A.: 1972a, Astron. Tzirkular, No. 683 (in Russian).

Teifel, V. G. and Kharitonova, G. A.: 1972b, Astron. Tzirkular, No. 735 (in Russian).

Teifel, V. G. and Kharitonova, G. A.: 1974, Astron. Zh. 51, 167 (in Russian).

Texereau, J.: 1967, Sky Telesc. 33, 226.

Trafton, L. M.: 1967, Astrophys. J. 147, 765.

Vdovitchenko, V. D.: 1974, Astron. Vestnik, in press.

Wallace, L., Caldwell, J. J., and Savage, B. D.: 1972, Astrophys. J. 172, 755.

Westphal, J. A.: 1969, Astrophys. J. 157, 63.

Westphal, J. A.: 1971, private communication.

Wrixon, G. T. and Welch, W. J.: 1970, Icarus 13, 163.

Yerbury, M. J., Condon, J. J., and Jauncey, D. L.: 1971, Icarus 15, 459. 
Yerbury, M. J., Condon, J. J., and Jauncey, D. L.: 1973, Icarus 18, 177.

\section{DISCUSSION}

Gulkis: I have also analysed the microwave spectrum of Saturn and found a significantly larger ammonia abundance than reported in this paper. I believe the reason for the discrepancy is the fact that my analysis used the recent Cornell University data at $50 \mathrm{~cm}$ and $70 \mathrm{~cm}$ whereas the longest wavelength data used in your analysis was $21 \mathrm{~cm}$. 